\title{
A DYNAMIC CLAMP PROTOCOL TO ARTIFICIALLY MODIFY CELL
}

\section{CAPACITANCE}

\author{
Paul Pfeiffer $\mathbb{D}^{1,2}$, Federico José Barreda Tomás ${ }^{2,3}$, Jiameng Wu $\mathbb{D}^{2,4}$, Jan-Hendrik Schleimer ${ }^{1}{ }^{1,2}$, Imre

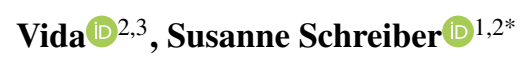 \\ ${ }^{1}$ Institute for Theoretical Biology, Humboldt-Universität zu Berlin, Philippstr. 13, Haus 4, 10115 Berlin, Germany \\ ${ }^{2}$ Bernstein Center for Computational Neuroscience, Humboldt-Universität zu Berlin, Philippstr. 13, Haus 6, 10115 \\ Berlin, Germany \\ ${ }^{3}$ Institute for Integrative Neuroanatomy, Charité-Universitätsmedizin Berlin, Corporate Member of Freie Universität \\ Berlin, Humboldt-Universität zu Berlin, and Berlin Institute of Health, Charitéplatz 1, 10117 Berlin, Germany \\ ${ }^{4}$ Einstein Center for Neurosciences Berlin, Berlin, Germany \\ *For correspondence: s.schreiber@hu-berlin.de
}

\begin{abstract}
Abstract - Dynamics of excitable cells and networks depend on the membrane time constant, set by membrane resistance and capacitance. Whereas pharmacological and genetic manipulations of ionic conductances are routine in electrophysiology, experimental control over capacitance remains a challenge. Here, we present capacitance clamp, an approach that allows to mimic a modified capacitance in biological neurons via an unconventional application of the dynamic clamp technique. We first demonstrate the feasibility to quantitatively modulate capacitance in a mathematical neuron model and then confirm the functionality of capacitance clamp in in vitro experiments in granule cells of rodent dentate gyrus with up to threefold virtual capacitance changes. Clamping of capacitance thus constitutes a novel technique to probe and decipher mechanisms of neuronal signaling in ways that were so far inaccessible to experimental electrophysiology.
\end{abstract}




\section{Introduction}

Membrane capacitance is a major biophysical parameter in neurons and other excitable cells, which determines how fast the membrane potential changes in response to a current [1, 2]. How capacitance impacts electrical signaling and neuronal processing, however, can rarely be observed experimentally, because its value appears to be constant for most membranes, around $1.0 \mathrm{uF} / \mathrm{cm} 2$ [3]. A prominent exception is the reduced capacitance of myelinated axons, which allows faster action potential propagation [4] and thereby crucially contributes to cognitive functions [5]. The effects of capacitance changes can, therefore, so far only be compared via mathematical simulations, where capacitance is simple to control. Such modeling, for example, suggests that the reduced membrane capacitance observed in human pyramidal cells can serve to increase synaptic efficacy [6, but see 7]. Nevertheless, experimental manipulation of capacitance remains challenging; in particular because changes in membrane area, thickness and lipid composition that affect capacitance might influence other membrane functions, such as the embedding of ion channels, with potentially unintended and uncontrolled consequences for electrical behavior. Here, we address this technical challenge by introducing capacitance clamp (CapClamp): an intracellular recording mode based on the dynamic clamp that emulates altered capacitance values in biological neurons [8, 9]. Via CapClamp, the voltage dynamics governed by the actual biophysics of a cell - active ion channels and synaptic inputs - can thus be flexibly probed under multiple "virtual" capacitance conditions, which provides precise experimental control over this hitherto inaccessible parameter.

In addition to the analysis of biological capacitance adaptations, control over capacitance offers a distinct way to probe cellular electrical dynamics. Capacitance has a unique temporal role, because its direct effects are restricted to the membrane time constant whilst leaving the steady state I-V curve unaltered. In this way, capacitance differs from leak conductance, the other determinant of the time constant, which also alters steady-state response amplitudes. For this reason, theoretical studies preferentially vary capacitance to investigate ion channel dynamics [10, 11] and qualitative switches (bifurcations) in neural excitability [12,13]. Furthermore, effects of an altered capacitance can be informative about more complex, time scale-related parameters like temperature or ion concentrations [14]. Such computational predictions, however, often rely on simplified neuron models, so a similar experimental control over capacitance would be desirable to test them in biological cells.

The proposed CapClamp alters capacitance in a virtual manner, combining the simplicity of computational control with the complex biophysics of a real neuron. It is inspired by the dynamic clamp technique, which has originally been developed to simulate the presence of additional conductances in a biological neuron relying on a fast feedback loop between intracellular recording and a computational model [8, 9, 15, 16]. The precise control over virtual conductances enables electrophysiological experiments that are more difficult or even impossible with traditional pharmacological or genetic means [17,-22]. Here, we demonstrate how the dynamic clamp can be extended to virtual capacitance modifications by currents designed to speed up or slow down dynamics of the membrane potential. We derive a simple expression for these CapClamp currents, which can be applied in all excitable cells and only requires the experimenter to specify the original cell and the desired target capacitance. In an experiment based on a hardware-implemented 
RC circuit, we verify that the CapClamp indeed correctly modifies the time constant. Via numerical simulations, we confirm that a clamped model neuron exhibits the same pronounced changes of firing and spike shape as a control cell with an altered capacitance. For an experimental demonstration, we clamp the near-somatic capacitance of rat dentate gyrus granule cells and analyze how the induced local capacitance change affects their spiking behavior. Finally, we illustrate how the CapClamp can be used to probe signal integration, energy consumption and bifurcations of excitable cells in ways that so far were experimentally inaccessible.

\section{Results}

\subsection{Capacitance clamp: a dynamic clamp protocol to mimic capacitance changes}

Dynamic clamp relies on a fast feedback loop between an intracellular recording of a neuron and a computer that simulates virtual cellular or circuit components online. Originally, the dynamic clamp has been developed to study how a membrane conductance alters the neuron's voltage dynamics [8, 9]. In each recording interval (i.e. time interval between two voltage samplings), a digital model of the conductance receives the sampled membrane potential, updates the conductance state and sends the corresponding current value back to the amplifier. In this way, given a sufficiently high update rate $f_{\text {dyn }}$ (often $\geq 10 \mathrm{kHz}$ ), the current through the recording electrode accurately mimics the current through the modeled conductance and the dynamics of the neuron appear as if this conductance was physically present in the membrane.

Whereas conductances gate ionic currents across the membrane, the capacitance determines how fast these currents can change the membrane potential. Every altered membrane property that results in a modified capacitance value, such as membrane area, thickness or lipid composition, affects this rate of change of the membrane potential (Fig. 1]A). To artificially mimic a modified capacitance, we therefore first asked whether a dynamic clamp protocol with its fast feedback loop between voltage sampling and current injection could adjust the "speed" of a cell's membrane potential (Fig.11B). Using the current balance equation, the basic mathematical description of membrane voltage dynamics, we derived a capacitance clamp (CapClamp) scheme with a simple expression for the clamping current $I_{d y n}$ (see Methods),

$$
I_{d y n, i}=\frac{C_{c}-C_{t}}{C_{t}}\left(C_{c} \frac{V_{i}-V_{i-1}}{\Delta t}-I_{d y n, i-1}\right)
$$

which only requires the experimenter to measure the cell capacitance $C_{c}$ in order to set a new target capacitance $C_{t}$. In every recording interval $\Delta t=f_{\text {dyn }}^{-1}$, the CapClamp uses the measured cell capacitance value $C_{c}$ and the voltage derivative $\frac{V_{i}-V_{i-1}}{\Delta t}$ to estimate the present membrane current and then increases $\left(C_{t}<C_{c}\right)$ or decreases $\left(C_{c}<C_{t}\right)$ the net current by insertion of a correction current in the next time bin. In this way, despite a physically unaltered capacitance, the membrane potential changes faster or, respectively, slower - as if the clamped cell actually had the different capacitance $C_{t}$ selected by the experimenter. In the following, we will demonstrate the CapClamp in simulated 
A

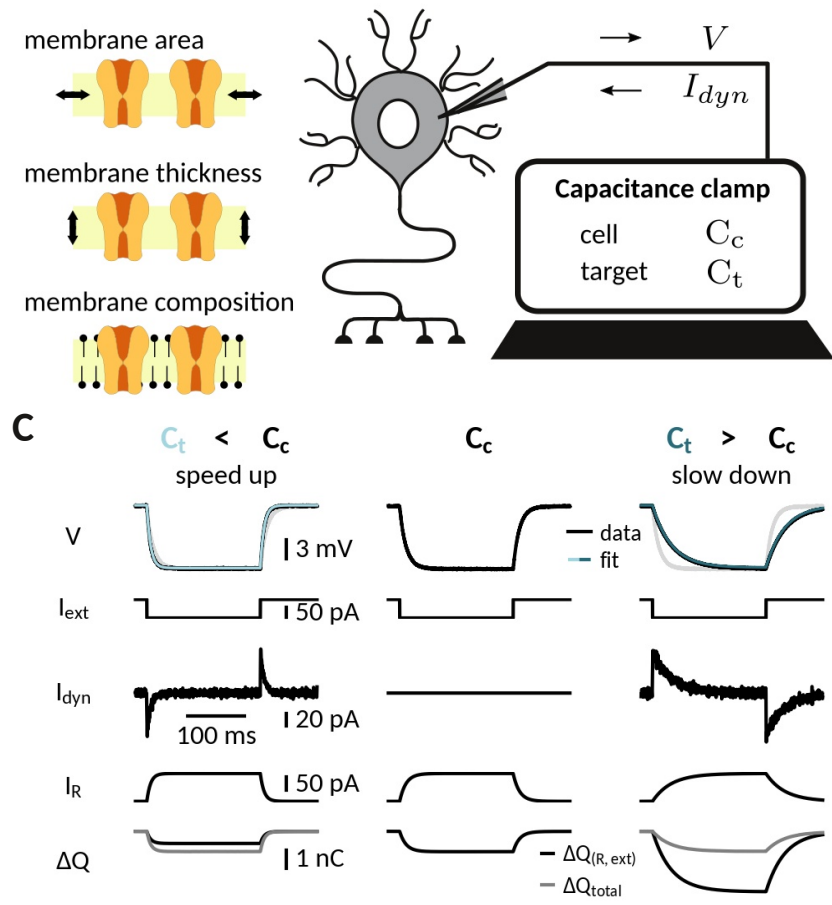

D
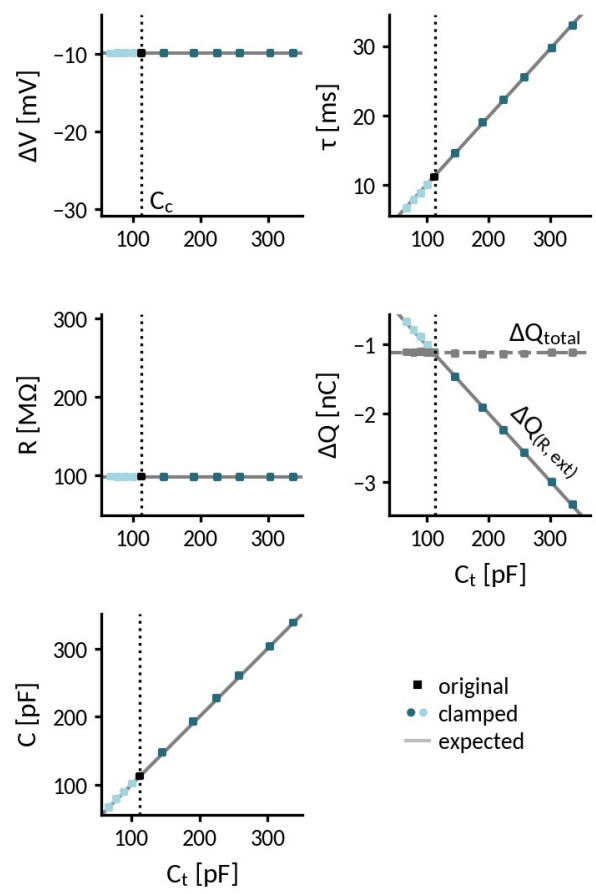

- original

- clamped

- expected

Figure 1: Adding or removing artificial capacitance via the CapClamp. A Physically, membrane capacitance varies with surface area, thickness and lipid composition B Virtual capacitance modification via the CapClamp is a form of dynamic clamp, a fast feedback between intracellular voltage sampling and computer-controlled current injection: given the measured cell capacitance $C_{c}$, the target capacitance $C_{t}$ and recorded membrane potentials, the computer calculates clamping currents required to mimic the desired change of capacitance. C Clamping a hardware-implemented model cell (RC circuit) at a decreased (left) or increased (right) capacitance leads to faster respectively slower charging of the "membrane potential" $V$ (top row, exp: black traces, exponential fit:light and dark blue) in response to a step current $I_{\text {ext }}$ (2nd row) due to the clamping currents $I_{\text {dyn }}$ (3rd row). As a result, the current through the resistance $I_{R}=-\frac{V}{R}$ (4th row) has a different profile and the apparently deposited charge $\Delta Q\left(R\right.$, ext) $=\int \mathrm{dt} I_{R}+I_{\text {ext }}$ (5th row, black) by the "cellular" transmembrane currents decreases, respectively, increases as expected for a capacitance change. The total deposited charge $\Delta Q_{\text {total }}$ (gray), taking into account the clamping currents, is the same in all three cases, because the physical capacitance did not change. D Measured time constant $\tau$, voltage responses $\Delta V$, resistance $R$, deposited charge $\Delta Q$ (apparent and total) and capacitance $C$ versus target capacitances.

and experimental scenarios with increasing complexity ranging from a passive $\mathrm{RC}$ circuit up to biological neurons with a spatially extended morphology.

\subsection{Clamping capacitance in a passive cell}

The simplest scenario to apply the CapClamp is a single compartment passive cell, equivalent to an RC circuit. In the absence of active conductances, the effects of a capacitance change can be precisely formulated: the capacitance $C$ sets the membrane time constant $\tau=R C$, determining how fast the membrane potential changes in response to a current. Note that, in contrast to the resistance $R$, the change in capacitance leaves the voltage amplitude of the steady-state response unaltered. To quantitatively confirm the effects of clamping capacitance and the ability of an exclusively temporal control, we measured time constant and capacitance of a clamped RC circuit in experiment and analyzed the temporal filtering properties of a modeled clamped circuit using mathematical analysis. 
To experimentally characterize a clamped passive cell, we implemented the CapClamp scheme in a dynamic clamp setup (see Methods) and recorded voltage responses to current pulses from the simplest possible model cell, i.e., a hardware implemented RC circuit, while clamping it at a range of target capacitances (Fig. 1]C). As expected for an RC circuit, the charging curve of the unclamped model cell was fit well by a single exponential, whose time constant $(\tau=11.1 \mathrm{~ms})$ and voltage amplitude $(\Delta V=-9.9 \mathrm{mV}$ ) allowed us to determine the circuit's resistance $R=99.4 \mathrm{M} \Omega$ and capacitance $C=112.3 \mathrm{pF}$. This capacitance value was then used as the cell capacitance $C_{c}$ input for the CapClamp. Clamped at a decreased capacitance, the time constant shortened $\left(C_{t}=67.4 \mathrm{pF}: \tau=6.6 \mathrm{~ms}\right)$ and at an increased capacitance, it lengthened ( $\left.C_{t}=336.9 \mathrm{pF}: \tau=33.0 \mathrm{~ms}\right)$, but in both cases the steady state voltage amplitude remained the same. Accordingly, the measured capacitance of the clamped circuit confirmed the chosen target capacitance for the whole tested range from a 0.6- up to a 3 -fold change with respect to the original capacitance (e.g. $C_{t}=67.4 \mathrm{pF}: C=67.5 \mathrm{pF}$; $C_{t}=336.9 \mathrm{pF}: C=338.1 \mathrm{pF}$ ), whereas the measured resistance remained constant (Fig. 11D).

As a consequence of the correctly transformed voltage response, the leak current in the clamped RC circuit also behaved as if the capacitance had changed. When the circuit was clamped, the leak current through the resistance, $I_{R}=\frac{V}{R}$, exhibited a shorter $\left(C_{t}<C_{c}\right)$ or longer $\left(C_{t}>C_{c}\right)$ transient until reaching steady state. As a consequence, the charge $\Delta Q\left(I_{R}, I_{\text {ext }}\right)$ deposited on the capacitance by the apparent "transmembrane" current, the sum of leak and external stimulus current, reduced $\left(C_{t}<C_{c}\right)$ or increased $\left(C_{t}>C_{c}\right)$ to the extent expected for an altered capacitance (Fig. 1 C). In contrast, the overall deposited charge $\Delta Q\left(I_{R}, I_{\mathrm{ext}}, I_{\mathrm{dyn}}\right)$, including the clamping current, was identical in the clamped and the original circuit, reflecting that the physical capacitance did not change. For the simple RC circuit considered here, the distinction between the clamping current and the intrinsic "cellular" currents might appear artificial, because all currents use the same charge carrier. In a biological neuron, however, this distinction becomes relevant, because the clamping currents through the recording electrode might rely on other charge carriers (depending on the used intracellular solution) than the cellular currents governed by multiple ion selective channel types.

For more complex stimuli than a simple current pulse, the temporal filtering properties of a clamped membrane determine how well the CapClamp mimics the chosen capacitance change. To generally assess these filtering properties, we analytically derived the frequency-dependent impedance of a modeled clamped RC circuit using linear control theory (Fig. 1 - suppl. 1 A, see Appendix. The derived impedance profiles confirmed the experimentally observed altered time constants. For example, an RC circuit clamped at an increased capacitance further attenuated non-zero frequencies reflecting its longer time constant. Overall, impedance amplitudes of a clamped RC and the corresponding target circuit fit well up to a tenth of the dynamic clamp frequency $f_{d y n}$, that is up to $\approx 2 \mathrm{kHz}$ for a $20 \mathrm{kHz}$ dynamic clamp system as used here (Fig. 1 - suppl. 1 B and C). As high frequencies are heavily attenuated by the low pass filter of a cell's membrane, these differences lead to relatively small deviations in the voltage responses. The mathematical analysis thus suggests that for a fast dynamic clamp system ( $>20 \mathrm{kHz}$ ), the CapClamp is expected to work well for most stimuli with time scales in the physiological range. 
A

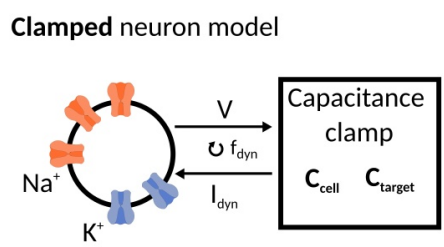

Control neuron model

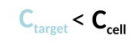<smiles>c1ccccc1</smiles>

with decreased cell capacitance e.g. reduced membrane area<smiles>C1C[As]C[As]C[As]C[As]1</smiles>

with increased cell capacitance e.g. enlarged membrane area

\section{C}

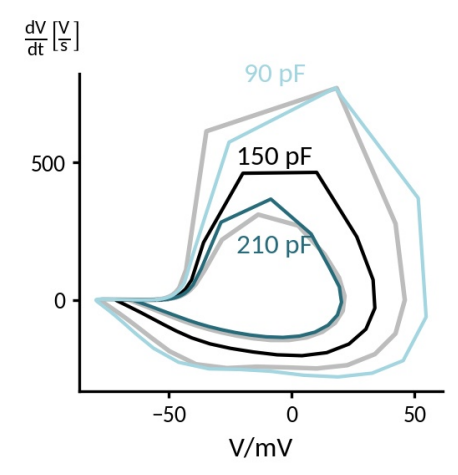

B
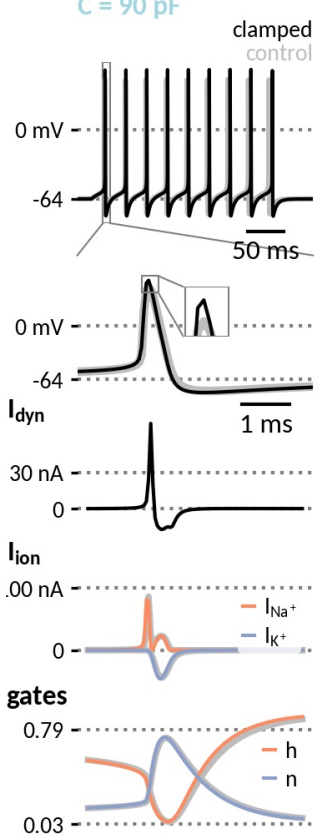

D
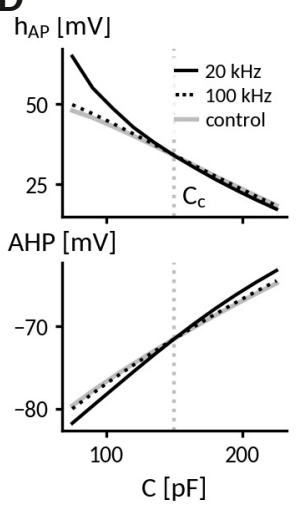

$\mathrm{C}=150 \mathrm{p}$
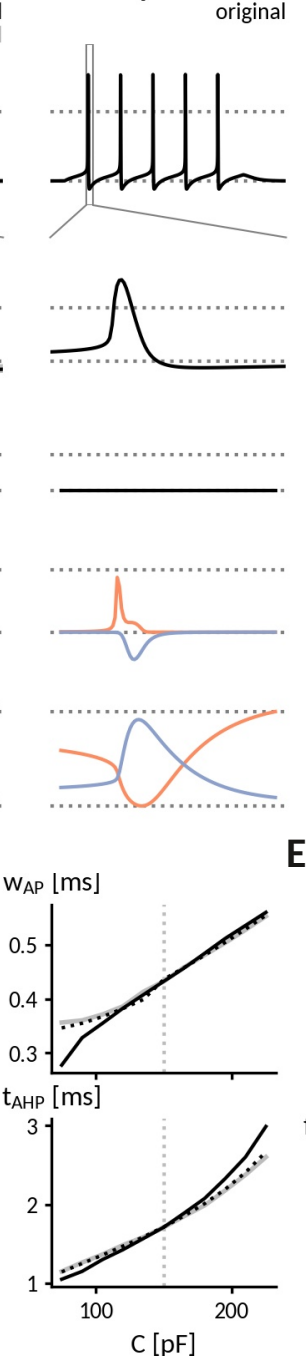

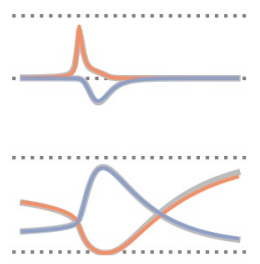

E
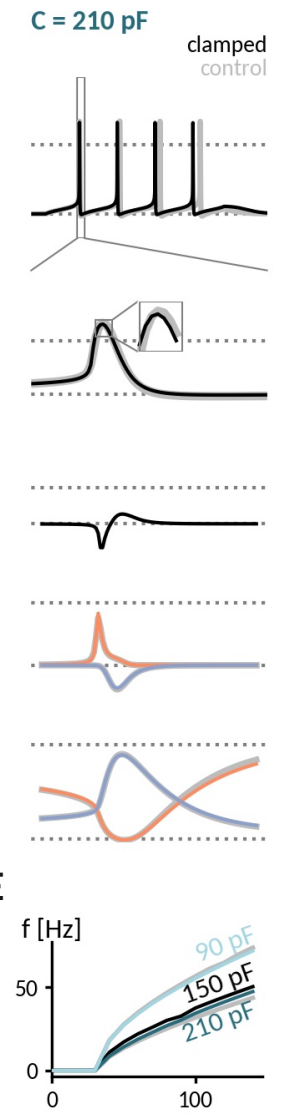
I [pA]

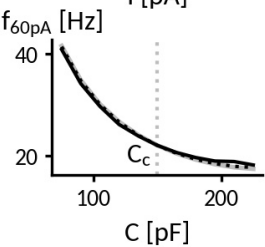

Figure 2: Simulation of the capacitance clamp in a conductance based neuron model. A Neurons coupled to the CapClamp are compared with control neurons with an altered capacitance (depicted as a difference in membrane area). B Spiking at 0.6-fold decreased $(90 \mathrm{pF})$, original $(150 \mathrm{pF})$ and 1.4-fold increased capacitance $(210 \mathrm{pF})$ with from top to bottom: spike shape, dynamic clamp current, ionic currents $\left(\mathrm{Na}^{+}, \mathrm{K}^{+}\right.$) and gating states ( $\mathrm{h}$ : sodium inactivation gate, $\mathrm{n}$ : potassium activation gate). Clamped and original traces in black or color, control in gray. C Comparison of spike shapes in the $\mathrm{V}-\frac{\mathrm{d} V}{\mathrm{dt}}$-plane (black: original, blues: clamped, gray: control). D Comparison of spike amplitude $h_{\mathrm{AP}}$ (top left), spike width $w_{\mathrm{AP}}$ (top right), after hyperpolarization amplitude AHP (lower left) and timing $t_{\mathrm{AHP}}$ (lower right) across different capacitances with two dynamic clamp frequencies (solid: $20 \mathrm{kHz}$, dotted: $100 \mathrm{kHz}$, gray:control). E Top: Comparison of f-I curves for capacitances in B. Bottom: Comparison of firing frequency at $60 \mathrm{pA}$ across different capacitances with two dynamic clamp frequencies (solid: $20 \mathrm{kHz}$, dotted: $100 \mathrm{kHz}$, gray:control). 


\subsection{Simulation of the CapClamp in a biophysical neuron model}

In neurons with active spike-generating conductances, capacitance changes impact neuronal firing via the interplay of the altered membrane time constant and the gating kinetics of the channels involved. As gating dynamics can be in the sub-millisecond range, e.g. for transient sodium channels, the CapClamp is expected to require a sufficiently high dynamic clamp frequency to accurately reproduce changes of spike shape or firing rate. To understand these requirements and lay the ground for investigations of capacitance changes in biological neurons, we simulated the CapClamp in a neuron model with biophysical channel dynamics and a single-compartment morphology (see Methods). The simulation allowed us to compare the firing of the clamped neuron to the expected firing at this modified capacitance. Specifically, we inspected the spiking responses to a depolarizing current for the original $150 \mathrm{pF}$, a decreased $90 \mathrm{pF}$ and an increased $210 \mathrm{pF}$ capacitance, for the latter two comparing clamped and expected dynamics (Fig. 22A). Capacitance changes exerted a notable influence on both firing frequency and spike shape, which was mostly well-captured by the simulated CapClamp (Tab. 11). When the capacitance was decreased to $90 \mathrm{pF}$, spiking frequency speeded up and action potentials had a larger peak amplitude, a decreased duration and an increased afterhyperpolarization (AHP). When the capacitance was increased to $210 \mathrm{pF}$, the effects were opposite: spiking frequency slowed down and action potentials had a smaller peak, an increased duration and a reduced AHP. At increased capacitances, spike amplitudes of the clamped neuron were larger than expected, a consequence of the limited tracking of the fast sodium current at the used dynamic clamp frequency (Fig. 2 2 B). Except for this brief overshoot, the CapClamp overall forced the membrane potential on the expected trajectory and correctly adjusted the resulting ionic currents and the gating variable dynamics of the active conductances, e.g. for a reduced capacitance of $90 \mathrm{pF}$, the sodium current became narrower in time and exhibited a second peak (Fig.2/A).

A subsequent comparison of simulated spiking for the whole range of tested target capacitances from $75 \mathrm{pF}$ to $225 \mathrm{pF}$ confirmed that the CapClamp reliably reproduced the main effects of a modified capacitance on spike shape (Fig. 2]C) and firing frequency (Fig. 2D). As expected, a crucial factor for a good quantitative fit is the dynamic clamp frequency observable differences at a $20 \mathrm{kHz}$ sampling frequency were strongly reduced for a sampling frequency of $100 \mathrm{kHz}$ (Fig. 2 2 and D). In this regard, the chosen neuron model is especially demanding because its rapid gating dynamics are fit to a fast spiking interneuron [23]. Taken together, our simulations show that capacitance impacts neuronal spiking from firing frequency to action potential shape and that the CapClamp is well-suited to study these effects.

Table 1: Firing frequency and spike shape in a biophysical neuron model for a decreased, the original and an increased capacitance, comparing simulations of an actually altered capacitance with the CapClamp. Values are shown as actual(clamped).

\begin{tabular}{ccccc}
\hline $\mathrm{C}(\mathrm{pF})$ & $\mathrm{f}(\mathrm{Hz})$ & $\mathrm{h}_{\mathrm{AP}}(\mathrm{mV})$ & $\mathrm{w}_{\mathrm{AP}}(\mathrm{ms})$ & $\mathrm{AHP}(\mathrm{mV})$ \\
\hline decreased 90 & $34.9(\mathbf{3 4 . 3})$ & $45.7(\mathbf{5 5 . 0})$ & $0.30(\mathbf{0 . 3 0})$ & $-77.8(-\mathbf{7 9 . 7})$ \\
original 150 & 22.1 & 33.9 & 0.39 & -71.5 \\
increased 210 & $17.8(\mathbf{1 8 . 9})$ & $21.4(\mathbf{2 0 . 1})$ & $0.48(\mathbf{0 . 4 8})$ & $-66.0(-\mathbf{6 4 . 7})$
\end{tabular}




\subsection{Experimental demonstration of the CapClamp in rat dentate gyrus granule cells}

Biological neurons differ from the simple "cells" considered so far, i.e. RC circuit and single compartment neuron model, in a major aspect: they can have complex morphologies, where the membrane potential varies between different compartments and membrane capacitance is distributed across the neuronal structure. As the CapClamp in contrast operates locally through the recording electrode, the emulated capacitance change is expected to be localized to the recorded compartment instead of affecting all compartments (see Methods). To demonstrate such localized capacitance changes and study their effects on neuronal spiking, we applied the CapClamp in in vitro patch-clamp recordings of rat dentate gyrus granule cells (DGGCs). Among morphologically complex cells, DGGCs appear well-suited to test the CapClamp, because their morphological structure, consisting of a central soma and one to four primary apical dendrites as shown in Figure 3] A [24], translates to a relatively compact electrotonic structure [25, 26].

\subsubsection{Measurement of local near-somatic capacitance}

Most capacitance measurements aim to provide an accurate estimate of the global capacitance of a neuron [1, 2]. To correctly infer the transmembrane and axial current, however, the CapClamp requires the local capacitance value of the compartment where the electrode is placed at. For the somatic DGGC recordings, we exploit that the current clamp step method - fitting charging curves via a sum of exponential terms - can also provide local capacitance information [1]. DGGC charging curves consisted of a slow $\left(\tau_{0}: 15.1 \pm 4.8 \mathrm{~ms}, R_{0}: 127 \pm 45 \mathrm{M} \Omega\right)$ and a fast $\left(\tau_{1}: 0.77 \pm 0.24 \mathrm{~ms}, R_{1}\right.$ : $35 \pm 15 \mathrm{M} \Omega$ ) component. Such a response can be understood in terms of a two compartment circuit consisting of a near compartment, comprising the patched soma and its surrounding, coupled to a far, mostly dendritic, compartment as depicted in Figure 3 A (for details on the mapping, see Methods). Importantly, the slow and fast components can be mapped to the corresponding five circuit parameters: near capacitance $C_{n}(21.0 \pm 9.4 \mathrm{pF})$, near resistance $R_{n}(854 \pm 394 \mathrm{M} \Omega)$, coupling resistance $R_{a}(53 \pm 20 \mathrm{M} \Omega)$, far capacitance $C_{f}(106 \pm 33 \mathrm{pF})$ and far resistance $R_{f}$ $(156 \pm 60 \mathrm{M} \Omega)$ (Fig. 3 C). Accordingly, this near-somatic capacitance $C_{n}$ represents the summed capacitance of the membrane area that is electrotonically close to the recording site and thus is the value that the CapClamp requires as input and should be able to modify.

\subsubsection{Altered near-somatic capacitance in DGGCs}

To confirm the localized effect of the CapClamp, we repeated the above capacitance measurement while clamping DGGCs at values ranging from 0.6 to 3 times the original near capacitance. Figure 3 B depicts how the charging of the membrane potential in an exemplary cell changed its shape in reaction to the clamp. Both slow and fast time constant lengthened with capacitances, whereas the associated resistances increased and decreased, respectively, such that their sum, the total input resistance (which is expected to be independent of capacitance), remained constant. These measured time constants and amplitudes matched the predicted ones for a two compartment circuit with a near capacitance at the chosen target values and all other circuit parameters at their original values. In a multicompartment simulation of a 
morphologically reconstructed DGGC, we could reproduce both the two compartment structure of DGGCs and the isolated modification of the near capacitance, further confirming the local control via the CapClamp.

Across 18 recorded cells, the CapClamp robustly altered DGGC charging curves and allowed to modify the charging time constants. Within the tested capacitance range, the slow time constant $\tau_{0}$ decreased by -0.8 (-1.0 to -0.6$) \mathrm{ms}$, median and interquartile range in parentheses, and increased up to 3.0 (2.4 to 3.9) ms, whereas the fast time constant $\tau_{1}$ changes ranged from -0.24 ( -0.29 to -0.20$) \mathrm{ms}$ up to 0.60 (0.36 to 0.86$) \mathrm{ms}$ (Fig. 3 D). To quantify how well these changes reflected an altered near capacitance, we evaluated the goodness of fit between the observed and expected time constants and resistances. In the majority of cells, R-squared values were close to 1 , indicating that the CapClamp induced the expected changes ( $\tau_{0}: 0.87$ (0.76 to 0.92$), R_{0}: 0.77$ (0.56 to 0.89$), \tau_{1}: 0.76$ (0.32 to 0.97$), R_{1}: 0.85$ $(0.75$ to 0.91$))$. The largest mismatches occurred for the fast time constant, especially at high capacitances, where the measured time constant was often shorter than predicted (Fig. 3 D). A small bias towards a shorter fast component is to be expected and also present in the multicompartment simulation, because this time constant was only about ten times longer than the recording interval of 50 us limiting its slow-down by the CapClamp currents. Larger deviations of $\tau_{1}$ however could not be reproduced in numerical simulations and likely result from other error sources, such as the difficulty of fitting this small and short time constant in the presence of noise or imprecise estimates of the original near capacitance (see Methods). Overall, in terms of circuit parameters, the capacitance measurements overall confirmed the targeted near capacitance change for 12 out of 18 cells within an average error of $10 \%$ (Fig. 3 E). Concluding, the CapClamp achieved an isolated change of the near-somatic capacitance in DGGCs and thereby allows to control the time constants of their passive voltage dynamics.

\subsubsection{Near-somatic capacitance governs action potential shape and firing frequency in dentate gyrus granule cells}

In neurons such as the recorded DGGCs, where the axon directly emerges from the soma, the ability to clamp the near-somatic capacitance provides control over the major capacitive load for the action potential generating site in the axon initial segment. Consequently, the CapClamp, although acting locally, is expected to impact action potential (AP) dynamics and excitability of a morphologically complex DGGC as demonstrated earlier for the simplified singlecompartment neuron model (Fig. 2). To illustrate how the CapClamp can thus be applied as as a novel probe to characterize neuronal firing, we compared spiking responses and f-I curves across near capacitances ranging from 0.6 to 3 times the original value, corresponding to a range from $10 \mathrm{pF}$ to $60 \mathrm{pF}$.

Clamping the near-somatic capacitance in DGGCs, we observed pronounced changes in the spiking response to depolarizing current step, clearly visible in the raw voltage traces (Fig. 4 A). The most apparent change was an altered AP shape (Fig. 4 B) - a continuous reduction of AP peak amplitude (from $61 \pm 9 \mathrm{mV}$ at $0.6 \mathrm{C}_{\mathrm{n}}$ to $24 \pm 16 \mathrm{mV}$ at $3 \mathrm{C}_{\mathrm{n}}$ ) and a simultaneous broadening of AP width (from $0.75 \pm 0.15 \mathrm{~ms}$ at $0.6 \mathrm{C}_{\mathrm{n}}$ to $1.30 \pm 0.40 \mathrm{~ms}$ at $3 \mathrm{C}_{\mathrm{n}}$ ) with increasing capacitance (Fig. 4 4 C and D). In addition, fast afterhyperpolarization (fAHP) was diminished and disappeared in the majority of cells after increasing capacitance (fAHP in $9 / 10$ cells at $0.6 \mathrm{C}_{n}$ and $2 / 10$ at $3 \mathrm{C}_{n}$ ). Importantly, the 
A

B

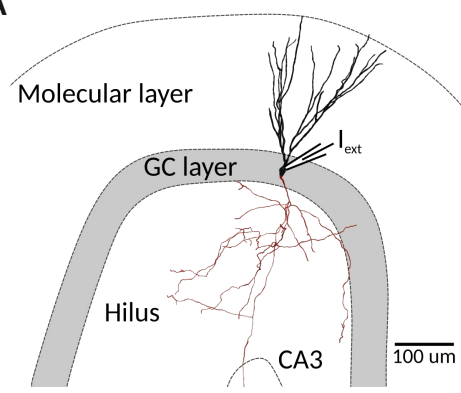

lext

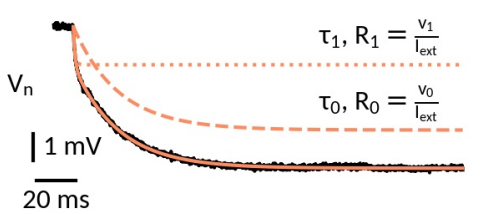

experiment: clamping near capacitance of a recorded DGGC

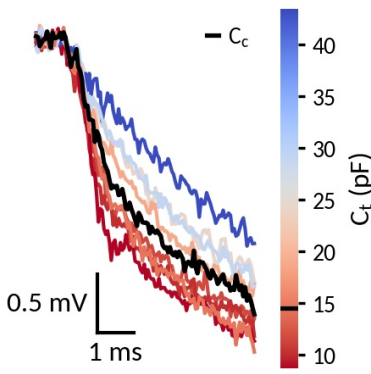

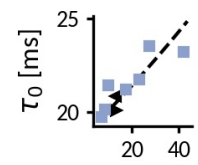

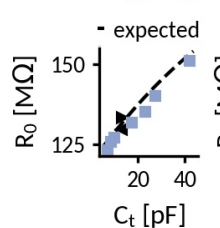

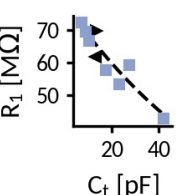
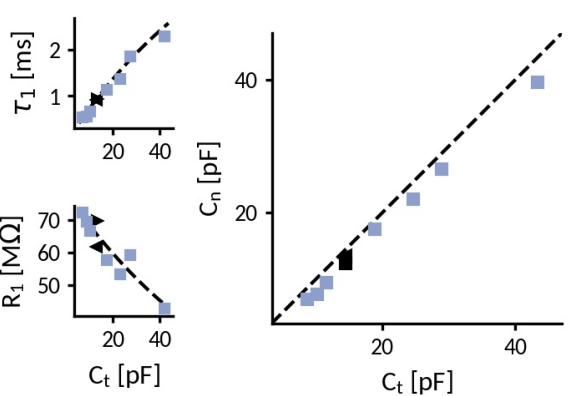

simulation: clamping near capacitance of a morphologically reconstructed DGGC
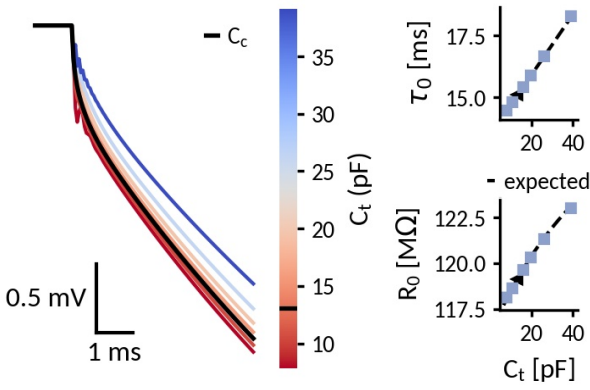

D
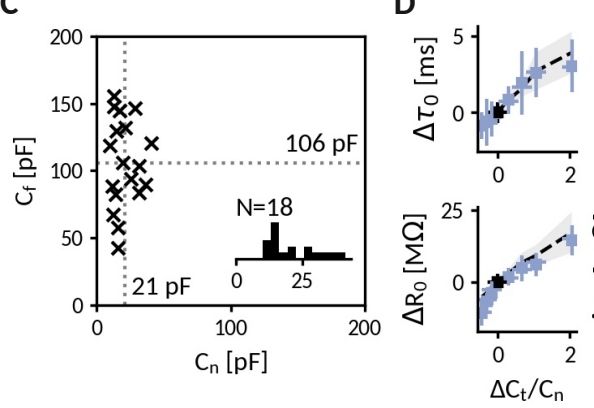
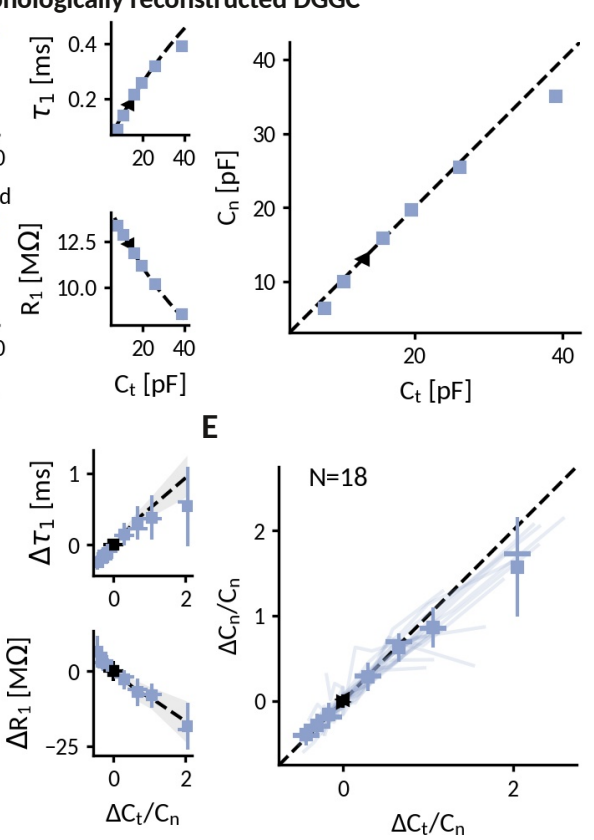
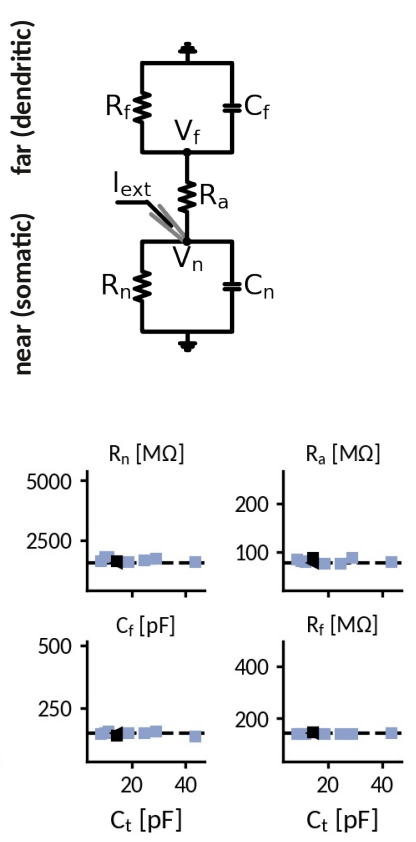

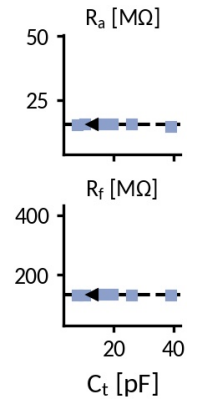

Cisplo
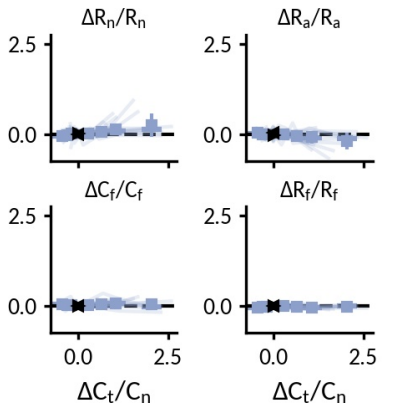

Figure 3: Clamping capacitance in rat dentate gyrus granule cells. A Morphology of a DGGC (left) and response to a hyperpolarizing current injected at the soma, fit via a sum of exponential terms with a slow $\tau_{0}, v_{0}$ and a fast component $\tau_{1}, v_{1}$ (middle), which can be mapped to two resistively coupled RC-circuits (right) with a near (somatic) compartment $C_{n}$ and $R_{n}$, resistive coupling $R_{a}$ and a far (dendritic) compartment $C_{f}$ and $R_{f}$. B Left: Voltage responses of a recorded (top) and a simulated morphologically-reconstructed (bottom) DGGC to a current pulse (exp: -27 pA, sim: -50 pA) clamped at 0.6- to 3-fold the cell's near capacitance (black: original, color: target near capacitances). Middle: Slow and fast components versus target capacitance. Right: Circuit parameters versus target capacitance. $\mathbf{4}, \mathbf{\bullet}$ : before and after clamping, blue square: clamped, dashed line: expected values. C Measured near $C_{n}$ and far $C_{f}$ capacitances for 18 DGGCs (gray dotted: mean). Inset: histogram of near capacitances. D Changes of slow and fast components in all recorded cells versus relative targeted change of near capacitance (squares: mean, horizontal line: median, vertical line: std, shaded area: std of expected changes). E Relative changes of circuit parameters versus relative targeted change of near capacitance. Legend same as in $D$ and individual cells shown with transparent blue lines. 
A decreased $\mathrm{C}=8.2 \mathrm{pF}$

original $\mathrm{C}=13.7 \mathrm{pF}$

increased $\mathrm{C}=41.1 \mathrm{pF}$

$f=10.5 \mathrm{~Hz}$

$\mathrm{f}=9.9 \mathrm{~Hz}$

$f=8.7 \mathrm{~Hz}$

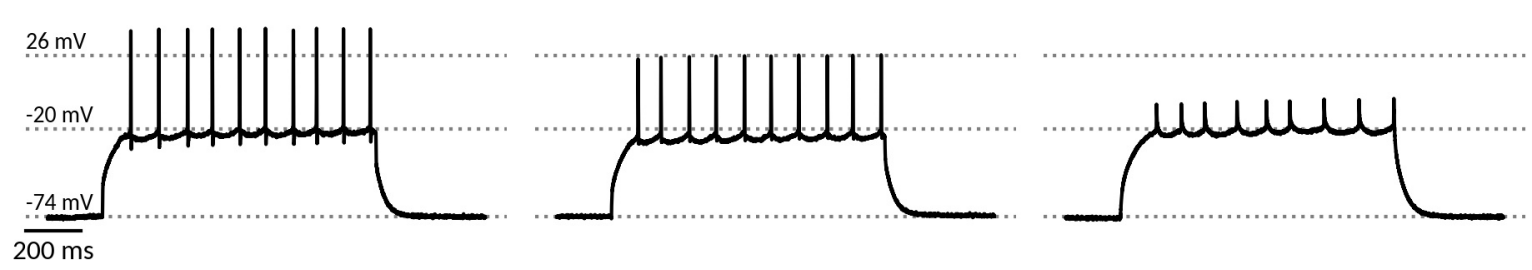

B
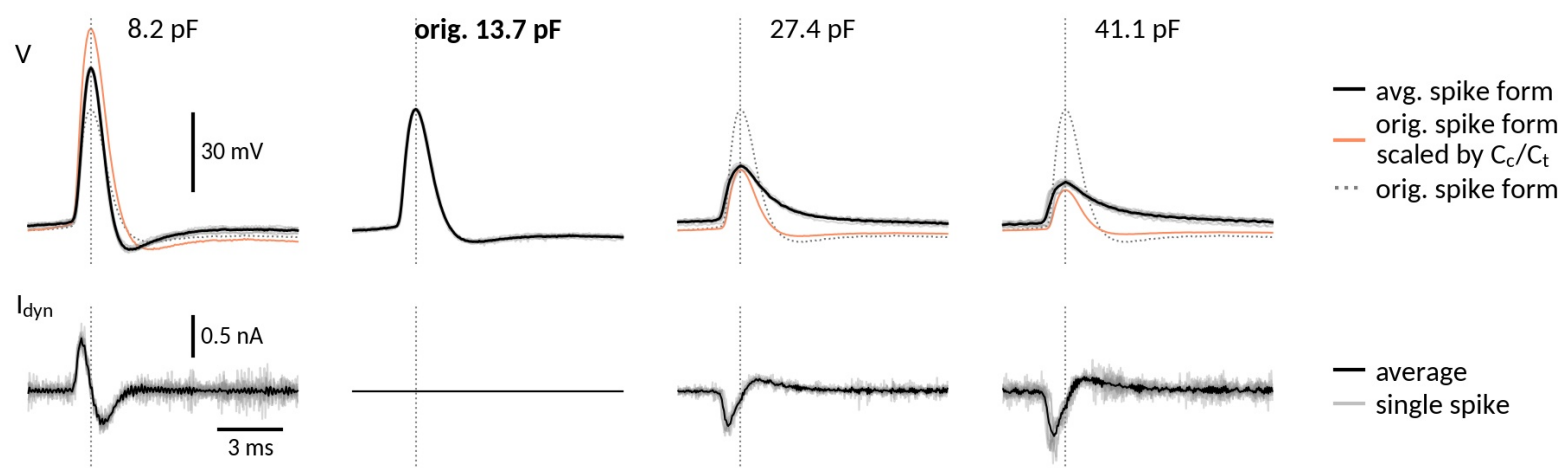

C

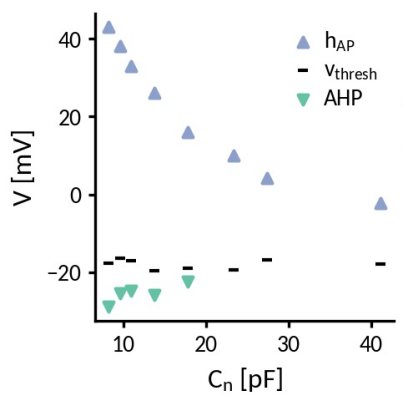

E

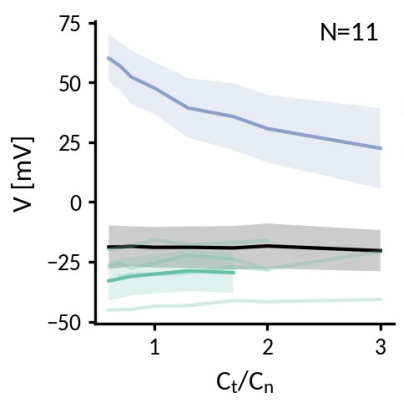

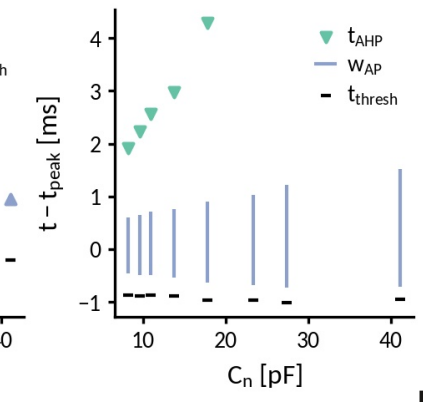

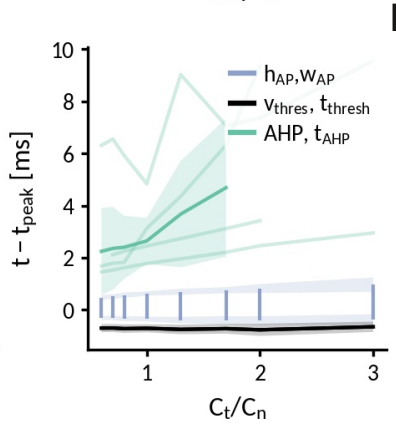

D
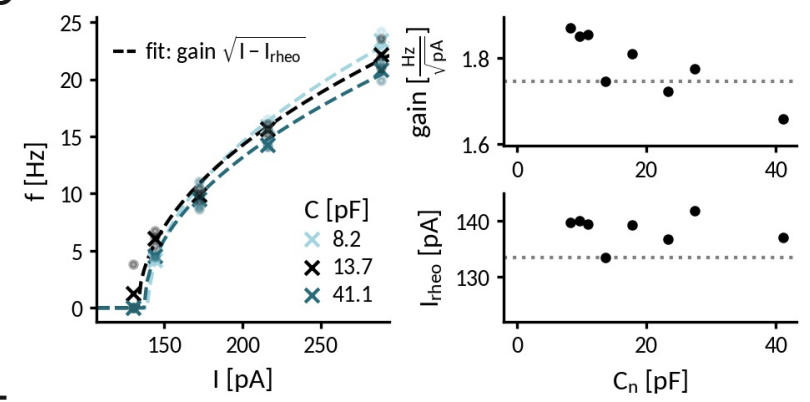

$\mathbf{F}$

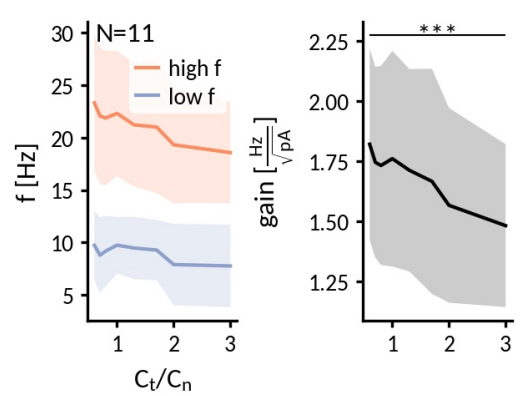

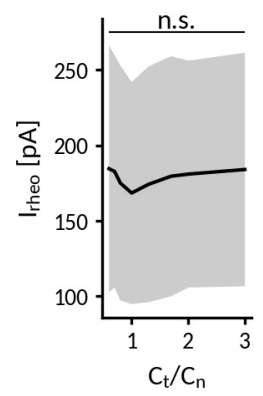

Figure 4: Repetitive spiking and action potential shapes in DGGCs clamped at different capacitances. A Spiking at decreased 0.6-fold (left), original (middle) and increased 3-fold (right) near capacitance $C_{n}$. B Spike shapes (top) and capacitance clamp currents (bottom) for increasing capacitances from 0.6 to 3 -fold of the original near capacitance (black: mean, light gray: single spikes, orange: expected spike shape for unaltered intrinsic currents, dotted: spike shape at original capacitance). C Comparison of spike shape (left) and temporal structure (right) across tested near capacitances. D Measured f-I at 0.6, 1 and 3-fold near capacitance with fit $f=$ gain $\sqrt{I-I_{\text {rheo }}}$ (dashed lines). Extracted gain and rheobase for all tested near capacitances (dotted line: values at original capacitance $13.7 \mathrm{pF}$ ). E Effect of capacitance changes on spike shape (left) and temporal structure (right) for all recorded DGGCs (solid: mean, shaded: std). To compare different cells, the capacitance is shown relative to the original near capacitance and spikes were compared at 1.2 fold of the cell's rheobase. F Effect of capacitance changes on firing frequency, low firing (blue) at 1.2 fold rheobase and high firing (red) at 2.0 fold rheobase (left), gain (middle) and rheobase (right) for all recorded DGGCs (solid: mean, shaded: std). 
observed disappearance of fAHP cannot be explained by increased capacitive filtering alone, which would decrease the its amplitude, but cannot abolish it. It thus demonstrates that the somatic capacitative load in DGGCs is able to influence the AP generating currents.

To illustrate the interplay of capacitance and the AP generating currents, we compared the observed spikes with hypothetical ones obtained by assuming unaltered currents with respect to the original capacitance (see Methods). Recorded and hypothetical spike shapes exhibited marked differences (Fig.4 B). At 0.6-fold decreased capacitances, for example, the recorded AP amplitude was significantly smaller than the hypothetical one (rec.: $61 \pm 9 \mathrm{mV}$, hyp.: 95 $\pm 18 \mathrm{mV}$, Wilcoxon signed-rank $p<0.001$ ), presumably reflecting a reduction of the driving force for the sodium current when the AP peak approaches the reversal potential of sodium. Furthermore, at 3-fold increased capacitance, as noted above the recorded spikes exhibited no fAHP in most cells while the hypothetical ones still did (fAHP rec: 2/10, hyp: 10/10) - potentially a result of a reduced activation of potassium channels due to lower AP amplitudes and/or earlier closing during the slowed AP repolarization. In contrast to driving force and gating dynamics, the channel kinetics, e.g. their activation curves, cannot be altered by capacitance. Correspondingly, the spike threshold, which reflects the voltage where sodium channels start to massively open, was not significantly correlated with near capacitance (Pearson correlation $\mathrm{r}=0.089, \mathrm{p}=0.42$ ). Taken together, our analysis indicates that an altered somatic capacitance affects both sodium and potassium currents in DGGCs.

Controlling the spike initiation zone, near-somatic capacitance also governed DGGC excitability. With increasing capacitance, DGGCs became less excitable and firing frequencies significantly decreased (Fig. $4 \mathrm{D}$ and F). From 0.6- to 3 -fold of the original near capacitance, the decrease was modest for low firing rates close to threshold (from $9.2 \pm 3.6$ $\mathrm{Hz}$ to $7.6 \pm 3.7 \mathrm{~Hz}$, Wilcoxon signed-rank $Z=47, p=0.024)$ and became more pronounced for high firing rates at the largest injected currents (from $22.2 \pm 7.0 \mathrm{~Hz}$ to $17.9 \pm 5.1 \mathrm{~Hz}, Z=55, p=0.001$ ). In this respect, the firing rate-current (fI) curves of the DGGCs resembled those obtained for the simulated neuron (compare Fig. 2D): the gain decreased for increased capacitances (from $1.74 \pm 0.45 \mathrm{~Hz} / \sqrt{\mathrm{pA}}$ at $0.6 C_{n}$ to $1.42 \pm 0.37 \mathrm{~Hz} / \sqrt{\mathrm{pA}}$ at $3 C_{n}, Z=55, p=0.001$ ), but the rheobase current remained relatively constant (from $182 \pm 77 \mathrm{pA}$ at $0.6 C_{n}$ to $179 \pm 75 \mathrm{pA}$ at $3 C_{n}, Z=12, p=0.13$ ). Overall, we conclude that a change of the near-somatic capacitance alone was sufficient to modify the input-output relationship of the recorded DGGCs.

\subsection{Applications of the CapClamp}

The CapClamp lends itself to either test hypotheses on the impact of capacitance or to exploit the control over the membrane time constant in order to to alter neuronal dynamics in informative ways. In the following, we briefly illustrate use cases of the CapClamp from these two fields, applying the technique to experimentally explore effects of capacitance on temporal integration, energetic costs of spiking and bifurcations. 


\subsubsection{Temporal integration}

A basic processing step in neuronal computation is temporal integration, the summation of time-separated synaptic inputs [27, 28]. An upper limit for temporal integration, at least in the absence of dedicated active channels, is set by the membrane time constant $\tau=R C$, which is directly proportional to the cell's capacitance. Hence, increasing the capacitance of a cell should make it a better integrator: if two inputs arrive separated in time, the cell's response to the second one should be higher than to the first one. Indeed, when we compared the responses of DGGCs to current pulse trains with different interstimulus intervals (ISI) clamped at decreased and increased near-somatic capacitances, a capacitance increase allowed the cell to better "sum" pulses at an ISI of $5 \mathrm{~ms}$ as apparent by the larger step sizes in the stair-like voltage response and the finally higher ratio of last to first pulse response. (Fig. 5 B). At an ISI of 50 $\mathrm{ms}$, in contrast, neither capacitance allowed temporal integration. The biological relevance of tailoring capacitance to temporal processing can, for example, be observed in auditory cells of the barn-owl, which have no dendrites to reduce capacitive and resistive load and hence shorten their time constant such that they can perform sub-millisecond coincidence detection [29].

\subsubsection{Energy consumption during spiking}

Action potentials are energetically expensive, because the required sodium and potassium ions have to be pumped back using ATP [20, 30]. The minimal amount of ionic charge required for an action potential is dictated by the capacitance as $Q=C \Delta V_{A P}$, suggesting that a smaller capacitance is energetically favorable. In order to gauge how capacitance affects charge deposit and energy consumption, we reexamined spike shapes for a fixed current input at different capacitances both in the simulated neuron and in the recorded DGGCs (Fig. 5 C and E). We found that despite a reduced amplitude at higher capacitances, these smaller spikes still required more depolarizating charge $Q_{+}=C \Delta V_{A P}$ (Fig. 5 $\mathrm{D}$ and F). In the model, we tested whether this depolarizing charge provided a reliable indication of the sodium charge $Q_{\mathrm{Na}^{+}}$, which finally determines pump activity and energy consumption (Fig. 5.D). The sodium charge exceeded the depolarizing charge, because sodium and potassium currents temporally overlap, but it increased in a similar manner with capacitance. Taken together, in the tested model and the recorded DGGCs, energy consumption per action potential appears to be reduced at smaller capacitances. In line with this observation, it has been reported that perineuronal nets could decrease membrane capacitance of fast-spiking interneurons, thereby facilitating high-frequency firing, while keeping energetic costs at bay [31].

\subsubsection{Neuronal bifurcations}

To optimally support neural processing, nerve cells exhibit qualitatively different response properties, which in some cases can be flexibly adapted to context. For example, neurons with class 2 firing (non-zero minimum frequency) can be switched to class 1 firing (arbitrarily low frequency) via neuromodulation [32, 33], transforming them from resonators to integrators. These qualitative differences in response and processing properties are linked to distinct spike generation mechanisms, whose corresponding excitability classes - as well as transitions between them - can be characterized 


\section{Temporal integration}

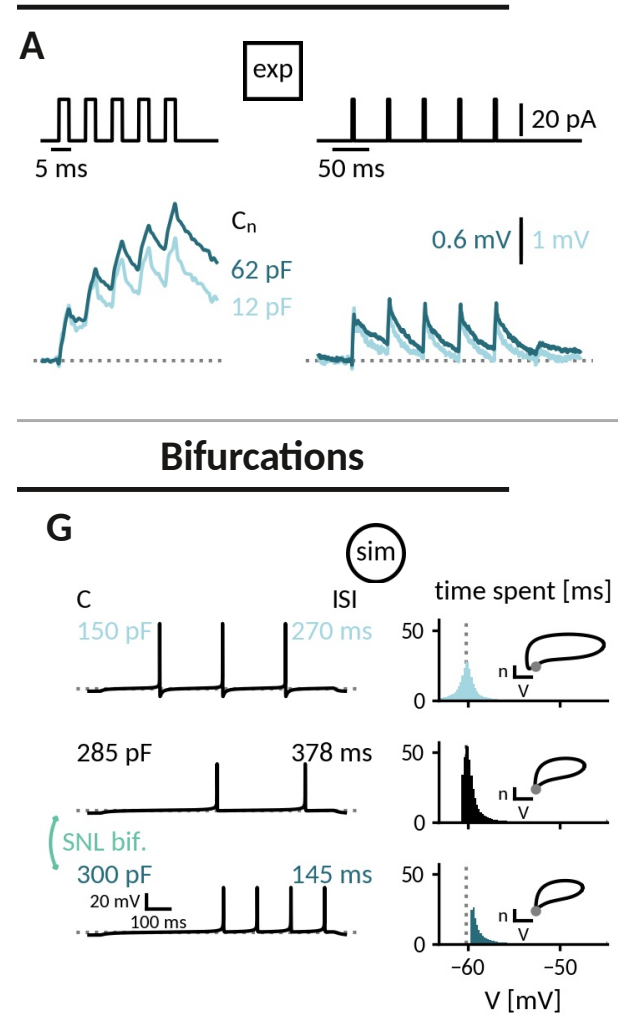

B

H

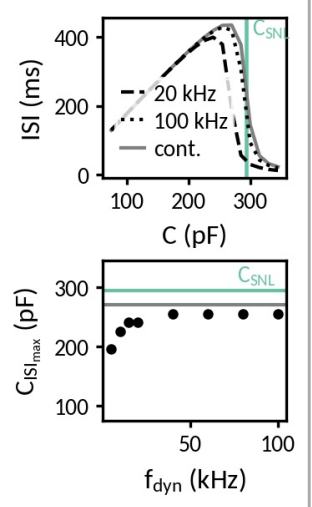

\section{Energetics of spiking}

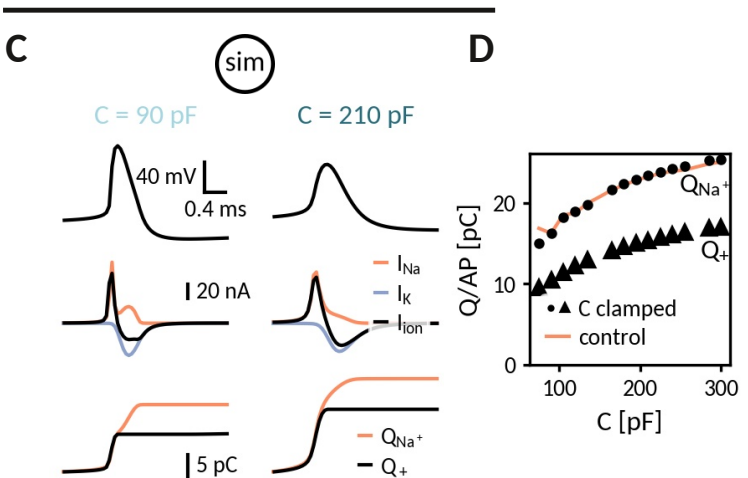

E

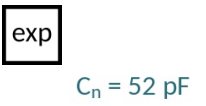

$\mathbf{F}$

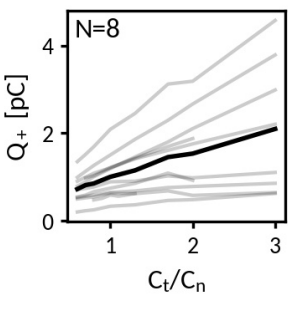

Figure 5: Applying the capacitance clamp to study neuronal signalling and physiology. Temporal integration: A Current pulses with interstimulus intervals of $5 \mathrm{~ms}$ and $50 \mathrm{~ms}$ (top) and voltage responses of an exemplary DGGC at a decreased (12 pF) and an increased $(62 \mathrm{pF})$ near capacitance (voltage scale adapted to first response height). B Ratio of fifth and first response as a measure of temporal integration for a 0.6 -fold decreased capacitance in comparison to a 3 -fold decreased one at $5 \mathrm{~ms}$ and $50 \mathrm{~ms}$ ISI. Energetics of spiking: C Spike shape (top), sodium, potassium and total ionic current (middle) and deposited sodium $Q_{\mathrm{Na}}$ as well as depolarizing $\mathrm{Q}_{+}$charge (bottom) in the Wang-Buzsaki neuron model for a $90 \mathrm{pF}$ and a $210 \mathrm{pF}$ capacitance. D Sodium $\mathrm{Q}_{\mathrm{Na}}$ and depolarizing $Q_{+}$charge per action potential versus capacitance (original: $150 \mathrm{pF}$, clamped: dot and triangle, control: gray). $\mathbf{E}$ Spike shape and depolarizing charge for a dentate gyrus granule cell clamped at decreased $10 \mathrm{pF}$ and increased $52 \mathrm{pF}$ capacitance. $\mathbf{F}$ Deposited depolarizing charge versus relative change of near capacitance in recorded DGGCs (black: mean, gray: individual cells). Studying bifurcations: G Left: In the Wang-Buzsaki neuron model, spiking slows down when increasing capacitance up to $285 \mathrm{pF}$, but a further small increase to $300 \mathrm{pF}$ abruptly doubles the firing frequency, a signature of a saddle-node-loop (SNL) bifurcation. Right: Histograms of the time spent at different membrane potentials between two spikes. Insets show the spike dynamics in the potassium gating $\mathrm{n}$-voltage $\mathrm{V}$ plane. H Capacitance clamp allows to induce the SNL bifurcation and locate the critical capacitance $\mathrm{C}_{\mathrm{SNL}}$. Top: Interspike interval against capacitance for simulated capacitance clamps at $20 \mathrm{kHz}$ (dashed) and $100 \mathrm{kHz}$ (dotted) dynamic clamp frequency versus the control simulation (gray) with indication of the expected critical capacitance $\mathrm{C}_{\mathrm{SNL}}$ (green). Bottom: Capacitance at maximal ISI against dynamic clamp frequency. 
by bifurcation analysis [34, 35]. Prior work recently highlighted the saddle-node loop (SNL) bifurcation, because it separates firing regimes with drastic differences in synchronization and can be induced by a wide array of physiological parameters, including temperature and extracellular potassium, as well as capacitance [13, 14]. We chose this transition and asked whether the CapClamp can correctly locate the SNL bifurcation and, therefore, provide an estimate where a neuron "positions itself" with respect to this critical switch.

To detect the SNL bifurcation in capacitance space, we looked for one of its footprints: frequency-doubling, a drastic increase of frequency for an incremental capacitance increase. In the simulated neuron, testing capacitances from $150 \mathrm{pF}$ up to $285 \mathrm{pF}$, the neuron fires at ever slower rates, but at $300 \mathrm{pF}$, the neuron indeed abruptly doubles its firing rate (Fig. 5 G). At this capacitance, the neuron fires faster, because the modified action potential switches to a trajectory, where it skips one half of the slow recharging after a spike (see the voltage histograms in Fig. 5 G). In the simulations, the CapClamp reproduced this frequency-doubling and, given a sufficiently fast dynamic clamp ( $>40 \mathrm{kHz}$ ), also correctly located the critical capacitance where the SNL bifurcations occurs (Fig. 5 H). In the recorded DGGCs, we found no evidence of frequency doubling in the tested capacitance range (Fig. $4 \mathrm{D}$ and F), indicating that the DGGCs are at a point in capacitance space further away from the SNL bifurcation.

\section{Discussion}

The dynamic clamp is a valuable tool in intracellular recordings to examine the diverse roles of ionic conductances in excitable cells [9, 15, 16, 36]. In this study, we introduced the capacitance clamp (CapClamp), an application of the dynamic clamp that allows to mimic a modified membrane capacitance in a biological neuron. Via simulations of a biophysical neuron model, we confirmed that the CapClamp correctly captures how capacitance affects spike shapes and firing frequency. In recordings of rat dentate gyrus granule cells, we further verified that the CapClamp could accurately control the capacitance of the recorded somatic compartments. Moreover, we clamped this near-somatic capacitance of DGGCs during spiking and found that, as predicted by our simulations, capacitance can modify the fI curve and alter the course of the spike generating currents. CapClamp can serve as a new probe to neuronal signaling, physiology and bifurcations. In the following, we highlight requirements for the CapClamp and discuss how this experimental control over capacitance can benefit the study of cellular electrical behavior.

\subsection{Precise, flexible and local control over capacitance in all excitable cells}

To our knowledge, the CapClamp is the first tool to experimentally study capacitance changes in a precise and flexible manner. The CapClamp owes its precision and flexibility to the virtual nature of the altered capacitance. In contrast, methods to physically modify the capacitance are affected by various undesired side effects. Dendritic pinching, decoupling dendrites from the soma, for instance greatly reduces membrane area and thereby capacitance, but also removes all dendritic conductances [37]. Capacitance alterations have also been reported after application of mefloquine, a drug binding to membrane phospholipids, but it also blocks gap junctions [38]. A notable exception is the recent 
demonstration of engineered polymer synthesis in neuronal cell membranes, which alters their capacitance, but not their input resistance [39]. In comparison, however, the CapClamp provides provides more accurate and dynamic control by allowing to test multiple selected capacitance values in a single cell (Fig. 3 and Fig. 47, while being significantly simpler to implement.

The CapClamp can be applied in every excitable cell. Here, we focused on neurons, but the proposed clamping currents can also be used to study capacitance changes in other cells, including for example heart cells [36, 40]. In particular, no prior knowledge about the ionic or external currents in the clamped cell is required, so that the capacitance can be clamped during any experimental (step current, ramp current, etc.) or during synaptic input. Furthermore, capacitance can be clamped in both electrotonically compact cells like oocytes [41] and non-compact cells like most neurons [26], although in the latter case the CapClamp is limited locally to the capacitance of the recorded compartment (Fig. 3). Consequently, the effects of clamping capacitance depend on the recording site and the cell's morphology. The soma, for example, represents the major capacitive load for spike generation in vertebrate neurons, where the axon predominantly emerges close to the soma (Fig. 44), but it is expected to exert less influence in neurons, where the axon comes out of the dendritic tree, a common feature of invertebrate neurons [42].

The major prerequisite to apply the CapClamp is a reliable capacitance measurement of the clamped compartment, which can be challenging, especially for electrotonically complex cells [1, 2]. An imprecise capacitance estimate leads to erroneous clamping currents, which increase high frequency noise for small errors and might even induce instabilities for larger errors. The measurement method presented for the recorded DGGCs, i.e. mapping the charging response to a two compartment circuit, could in principle be extended to cells with a larger number of compartments e.g. pyramidal cells [43, 44]. Yet, accurate multi-exponential fitting is demanding and the assumption of uniform membrane properties underlying the mapping is a simplification, shown to be violated in some cells, such as GABAergic interneurons [45]. As an alternative, measurement protocols could be exploited that inherently yield local capacitance estimates, including fast voltage ramps [1] or sampling of voltage responses to fast fluctuating currents [46]. Reliable capacitance measurements further allow to compare measured and target capacitance of the clamped cell, which serves as a first simple test to ensure the quality of the CapClamp.

\subsection{A CapClamp on every rig}

As a novel application of the established dynamic clamp technique, the CapClamp is an accessible and low-cost extension of a standard electrophysiology stack [15, 16]. For an existing dynamic clamp setup, the sole requirement is to implement the calculation of the clamping currents (see Eq. 5). Otherwise, multiple open source frameworks exist that only require a dedicated computer with a data acquisition card to enable the dynamic clamp in a conventional electrophysiology setup [47-53]. To facilitate the usage of the technique, we provide code for the CapClamp scheme in the RELACS and RTXI frameworks (see Appendix). 
In CapClamp recordings, as in all dynamic clamp applications, a high sampling frequency and accurate voltage monitoring are key [54]. Whether a sampling frequency is sufficiently high can be tested by assuring that the observed voltage dynamics e.g. the spike amplitudes are invariant when the sampling frequency is decreased from the maximal possible value [8]. For the simulated fast-spiking interneuron, we found a satisfactory clamp at a frequency of $20 \mathrm{kHz}$, which we expect to also be sufficient for most excitatory neurons, because they tend to have slower voltage dynamics [20]. In our single electrode recordings, we focused on careful electrode compensation to avoid electrode artifacts in the recorded voltages which would lead to incorrectly estimated membrane currents and eventually instabilities. To improve voltage monitoring, future applications could either apply active electrode compensation [55, 56] or resort to two electrode recordings, where current injection and voltage recordings are separated.

\subsection{Modifying capacitance as a probe for cellular electrical dynamics}

Via the CapClamp, experimenters can ask a question that was previously accessible only in theoretical work: What if capacitance was different? In contrast to the theoretical approach, the answers to this question do not have to rely on models of channel dynamics or other membrane properties, because the latter are provided by the biological cell itself [9]. Modifying capacitance with the CapClamp can serve either to investigate changes in this biophysical parameter or, more broadly, to alter the membrane time constant of a cell as a way to characterize its electrical dynamics.

\subsubsection{Understanding the role of capacitance}

The virtual capacitance changes induced by the CapClamp could serve to address two crucial questions about actual membrane biophysics: why capacitance appears to be biologically mostly constant [3] and how exceptions to this rule can facilitate or deter neuronal function [4, 6, 31, 57]. Capacitance is for example rarely tested for optimality - a common question in ion channel kinetics, which appear optimized for function and energy expenditure [20, 58]. Regarding energy consumption, our CapClamp experiments in DGGCs indicate that action potentials become energetically cheaper at lower capacitances (Fig. 5 E and F). Interestingly, reports of exceptional capacitance values mostly find reductions e.g. for myelinated axons $\left(C_{m} \approx 0.05 \mathrm{uF} / \mathrm{cm} 2\right.$ for a ten-fold wrapped myelin sheath, see [59]) or human pyramidal cells $\left(C_{m} \approx 0.5 \mathrm{uF} / \mathrm{cm} 2\right.$, see [6]) suggesting that indeed the metabolic cost of AP generation could have been a contributing factor to capacitance adaptations. Moreover, the recent hypothesis that perineuronal nets can reduce capacitance of interneurons in a similar way as myelination of axons suggests that capacitance adaptation could be more widespread in the brain than often assumed [31].

\subsubsection{Altering the membrane time constant}

A key contribution of the CapClamp is the isolated experimental control of the membrane time constant. Monitoring how the membrane potential dynamics change in response to such a perturbation of the time constant has been a theoretical tool to characterize a cell's electrical behavior [11, 13]. As an experimental analogue, the CapClamp could for example be used to further constrain and improve fitting of conductance-based neuron models [11, 60, 61]. Furthermore, the 
CapClamp can be used to identify capacitance values where qualitative changes of activity occur (bifurcations, see Fig. [5] G and $\mathrm{H}$ ), such as a switch to bistable firing [13, 14]. If a neuron is found to be close in capacitance/membrane time constant space to such a critical switch, this can have important implications for infrared [62, 63] and ultrasonic [64, 65] stimulation of neural activity, whose effects are assumed to rely on rapid alteration of the capacitance, as well as for other perturbations such as changes of temperature [13] or extracellular potassium [14], which have similar temporal effects as capacitance. Finally, the broad impact of the time constant on firing frequency and spike shape could be used to examine activity-dependent physiological processes such as ion concentration dynamics [14] or calcium controlled channel homeostasis [66-68].

\subsection{Conclusion}

Taken together, the presented CapClamp enables an accurate and flexible control over capacitance in biological neurons, a basic determinant of cellular excitability, that so far has been inaccessible in experiment. We expect that the CapClamp will, therefore, broaden and enrich the electrophysiological study of neurons and other excitable cells. With expanding techniques to sense and manipulate neural activity, the combination of modeling and targeted closed-loop feedback that underlies the CapClamp (and more generally the dynamic clamp [69]) will further unlock experimental control over other previously inaccessible aspects of single neuron [70-72] and network dynamics [73, 74]. 


\section{Materials and Methods}

\subsection{Derivation of the CapClamp current}

In order to derive a dynamic clamp feedback scheme for the CapClamp, we compare the actual membrane potential dynamics at the original capacitance $C_{c}$ with the target dynamics at the chosen capacitance $C_{t}$. The actual dynamics of the cell, which for the moment is assumed to be isopotential, is given by the current-balance equation of a single compartment

$$
\frac{\mathrm{d} V}{\mathrm{~d} t}=\frac{I(V, t)+I_{d y n}(t)}{C_{c}},
$$

with capacitance $C_{c}$, membrane currents $I(V, t)$ (comprising all ionic and synaptic currents, as well as external stimuli) and the dynamic clamp current $I_{d y n}(t)$. Note that ionic and synaptic contributions to the membrane currents $I(V, t)$ are voltage-dependent, both with respect to driving force and gating dynamics, so that a voltage trajectory governed by a different capacitance also leads to a modified shape of the membrane currents. In the target dynamics, the dynamic clamp current is absent and the capacitance is modified to the desired value

$$
\frac{\mathrm{d} V}{\mathrm{~d} t}=\frac{I(V, t)}{C_{t}}
$$

Both membrane potential trajectories would coincide, if we chose a dynamic clamp current such that the right-hand sides of actual (Eq.2) and target dynamics (Eq. 3) become identical,

$$
I_{d y n}(t)=\frac{C_{c}-C_{t}}{C_{t}} I(V, t) .
$$

Generally, an exact model for the membrane currents $I(V, t)$ will not be available, as it would require knowledge about all active conductances and incoming synaptic inputs. Instead, the membrane current can be estimated from the stream of incoming voltage data using the discrete version of Equation 2

$$
I\left(V_{i-1}, t_{i-1}\right) \approx C_{c} \frac{V_{i}-V_{i-1}}{\Delta t}-I_{d y n, i-1}
$$

where $\Delta t$ is the sampling interva! A prerequisite is the measurement of the cell capacitance $C_{c}$. Furthermore, for the estimation to be accurate, the sampling interval needs to be shorter than the fastest time scales of changes in the

\footnotetext{
${ }^{1}$ The indexing in Equation 5 assumes a voltage sampling $V_{i}=V(i \Delta t)$ and a current injection $I_{d y n, i}=I_{d y n}(i \Delta t)$. However, sampling can take a non-negligible amount of time, so that depending on the sampling system the currently available voltage actually represents the voltage from the previous cycle $V_{i}=V((i-1) \Delta t)$. In this case, for a correct estimation of the membrane currents, the dynamic clamp current index has to be shifted correspondingly,
}

$$
I_{d y n, i}=\frac{C_{c}-C_{t}}{C_{t}}\left(C_{c} \frac{V_{i}-V_{i-1}}{\Delta t}-I_{d y n, i-2}\right) .
$$


membrane currents e.g. sodium gating time constants. With this estimated membrane current, the complete expression for the CapClamp current reads

$$
I_{d y n, i}=\frac{C_{c}-C_{t}}{C_{t}}\left(C_{c} \frac{V_{i}-V_{i-1}}{\Delta t}-I_{d y n, i-1}\right) .
$$

The above derivation assumes that the cell is isopotential. In the case of an electrotonically non-compact cell, the steps are identical, but the cell capacitance $C_{c}$ has to be replaced by the capacitance of the compartment where the recording electrode is located. Consequently, in a non-isopotential neuron, the mimicked capacitance modification is restricted to the compartment at the tip of the recording electrode - a constraint known as the space clamp that is shared by all clamping techniques [15, 75].

\subsection{Capacitance measurements}

To apply the CapClamp, a prerequisite is to measure the capacitance of the recorded local compartment. Here, we use the current clamp protocol, which estimates the capacitance from the voltage response to a current step with amplitude $I_{\text {ext }}$,

$$
V(t)=\sum_{i} v_{i}\left(1-e^{-\frac{t}{\tau_{i}}}\right)=I_{e x t} \sum_{i} R_{i}\left(1-e^{-\frac{t}{\tau_{i}}}\right),
$$

where an ordering in terms of these time scales is assumed i.e. $\tau_{0}>\tau 1>\ldots$ Depending on the morphology, this sum can have a large number of components [76], but in practice often only two or three components can be reliably extracted. As described in Golowasch et al. [1], the slowest component $\tau_{0}$ is the membrane time constant and allows to infer the total capacitance of a neuron by $C=\frac{\tau_{0}}{R_{0}}=\frac{\tau_{0}}{v_{0}} I_{\text {ext }}$. In the case of an isopotential cell, the membrane time constant is the only component in the charging curve and the total capacitance can be used for the CapClamp.

\subsubsection{Measurement of near capacitance}

For the case of two components $\tau_{0}, R_{0}$ and $\tau_{1}, R_{1}$ in the charging curve (Eq. 6), an equivalent two compartment circuit can be identified comprising a near compartment with capacitance $C_{n}$ and resistance $R_{n}$ connected via a coupling resistance $R_{a}$ to a far compartment with capacitance $C_{f}$ and resistance $R_{f}$ [1, Appendix A]. With the additional assumption of a uniform membrane time constant $\tau_{m}=R_{n} C_{n}=R_{f} C_{f}$, the fitted two components can be mapped to the values of these five circuit parameters, which in particular provides the near capacitance $C_{n}$ required for the CapClamp (for the full mapping see [Appendix][Mapping a charging curve with two components to a two compartment circuit])

$$
C_{n}=\frac{\tau_{0} \tau_{1}}{\tau_{1} R_{0}+\tau_{0} R_{1}}
$$

When the capacitance is subsequently clamped to a k-fold different value, $C_{t}=k C_{n}$, the uniformity assumption has to be correspondingly adjusted to $R_{n} C_{n}=k R_{f} C_{f}$. 


\subsection{CapClamp in dentate gyrus granule cells}

\subsubsection{Electrophysiology}

Acute brain slices were produced as described earlier [77]. Briefly, rats were anesthetized (3\% Isoflurane, Abbott, Wiesbaden, Germany) and then decapitated. Brains were removed quickly and transferred to carbogenated $\left(95 \% \mathrm{O}_{2} /\right.$ $5 \% \mathrm{CO}_{2}$ ) ice-cold sucrose-ACSF containing (in $\mathrm{mM}$ ): $87 \mathrm{NaCl}, 2.5 \mathrm{KCl}, 25 \mathrm{NaHCO}_{3}, 1.25 \mathrm{NaH}_{2} \mathrm{PO}_{4}, 25$ glucose, 75 sucrose, $7 \mathrm{MgCl}_{2}, 0.5 \mathrm{CaCl}_{2}, 1$ Na-pyruvate, 1 ascorbic acid. Horizontal brain slices of $300 \mu \mathrm{m}$ thickness were cut using a Vibratome (VT1200 S, Leica, Wetzlar, Germany). Hippocampal tissue slices, were collected and placed in a submerged holding chamber filled with carbogenated sucrose ACSF at $32-34{ }^{\circ} \mathrm{C}$ for 30 minutes and then at room temperature for 15 minutes before recording. Experiments were alternated between left and right hemisphere slices to prevent bias due to slice condition.

For recording, slices were transferred to a submerged chamber and superfused with pre-warmed, carbogenated ACSF containing (in mM): $125 \mathrm{NaCl}, 2.5 \mathrm{KCl}, 25 \mathrm{NaHCO}_{3}, 1.25 \mathrm{NaH}_{2} \mathrm{PO}_{4}, 25$ glucose, $1 \mathrm{MgCl}_{2}, 2 \mathrm{CaCl}_{2}, 1 \mathrm{Na}$-pyruvate, 1 ascorbic acid. The bath temperature was set to $32-34^{\circ} \mathrm{C}$ with a perfusion rate of $12-13 \mathrm{ml} / \mathrm{min}$. Slices were visualized using an upright microscope (AxioScope; Zeiss) equipped with infrared differential inference contrast optics and a digital camera (Retiga EX QImaging CCD, Teledyne Photometrics, AZ, USA). Granule cells from the DG were chosen based on their anatomical location within the hilus as well as their morphological appearance.

Whole-cell patch-clamp electrodes were produced from borosilicate glass capillaries (outer diameter $2 \mathrm{~mm}$, inner diameter $1 \mathrm{~mm}$, Hilgenberg, Germany) using a horizontal puller (P-97, Sutter Instruments, CA, USA) and filled with an intracellular solution consisting of (in mM): K-gluconate 130, $\mathrm{KCl}$ 10, HEPES 10, EGTA 10, $\mathrm{MgCl}_{2} 2, \mathrm{Na}_{2} \mathrm{ATP} 2$, $\mathrm{Na}_{2}$ GTP $0.3, \mathrm{Na}_{2}$ Creatine 1 and $0.1 \%$ biocytin (adjusted to $\mathrm{pH} 7.3$ and $315 \mathrm{mOsm}$ ), giving a series resistance of 2.5-4 M $\Omega$. All recordings were performed with a SEC LX10 amplifier (npi electronic, Germany), filtered online at $20 \mathrm{kHz}$ with the built-in Bessel filter, and digitized at $20 \mathrm{kHz}$ (National Instruments, UK). Following breakthrough into whole-cell configuration, we adjusted the bridge and capacitance compensation before switching to the dynamic clamp mode for recording. Cells were excluded if resting membrane potential was more depolarized than $-45 \mathrm{mV}$. The liquid junction potential was not corrected.

Neuronal visualization and immunohistochemistry Following recording, selected cells were immersion fixed in $4 \%$ paraformaldehyde (PFA) in $0.1 \mathrm{M}$ phosphate buffer (PB, pH 7.4) at $4^{\circ} \mathrm{C}$ for 24 - 48 hours, slices were then transferred to fresh PB. Prior to immunohistochemical processing, slices were rinsed in PB, followed by PB buffered saline (PBS, $0.9 \% \mathrm{NaCl}$ ). Slices were then rinsed in PBS and incubated in a fluorescent-conjugated streptavidin (Alexa Fluor-647, 1:1000, Invitrogen, UK) in PBS solution containing 3\% NGS, $0.1 \%$ TritonX-100 and $0.05 \%$ NaN3 for 24 hours at $4^{\circ} \mathrm{C}$. Slices were rinsed in PBS and then desalted in PB before being mounted (Fluoromount-G, Southern Biotech) on 300 $\mu \mathrm{m}$ thick metal spacers, cover-slipped, sealed, and stored at $4^{\circ} \mathrm{C}$ prior to imaging. 
Confocal imaging and reconstruction DGGCs were imaged on a laser scanning confocal microscope (FV1000, Olympus, Japan). First, a low magnification (4x air immersion, Olympus, Japan) overview image was taken to confirm the cellular type and localization to the DG, then high resolution z-stacks were made with a 30x silicone immersion lens (N.A. 1.05, UPlanSApo, Olympus) over the whole extent of the cell (1 $\mu \mathrm{m}$ axial steps). Images were reconstructed offline using the FIJI software package (imagej.net) and Neutube (neutracing.com) [78]. Image stacks were stitched in FIJI, then the cells were reconstructed and volume filled using Neutube.

Dynamic clamp setup Data acquisition and dynamic clamp loop were controlled by RELACS, V0.9.8, RRID:SCR_017280 using a dedicated computer with a Linux-based real time operating system (rtai.org). The sampling frequency was set to $20 \mathrm{kHz}$ and the recordings were performed in discontinuous current clamp with a duty time of $16.5 \mu$ s. We implemented a CapClamp procedure for RELACS that allows the user to online specify the measured capacitance $C_{c}$ and the desired target capacitance $C_{t}$ (for documentation and install instruction see Appendix.

\subsubsection{Online measurement of capacitance}

For the online measurement of the local capacitance, DGGCs were subjected to twenty hyperpolarizing pulses of $200 \mathrm{~ms}$ length with $400 \mathrm{~ms}$ pauses and an amplitude chosen to produce a response of $-5 \mathrm{mV}$ in order to minimize interference from active ionic currents. Responses were averaged and the resulting mean trajectory was fit with a sum of exponentials using the Levenberg-Marquardt method from the python library scipy [79]. Fits were performed with one, two and three components and were compared via the F-statistic [80]. In all recorded DGGCs, the two component fit was significantly better than the one exponential fit $(p<0.05,18 / 18)$, whereas no cell exhibited a significant third component $(p<0.05,0 / 18)$. Finally, the extracted two components were mapped to a two compartment circuit as explained above and the near capacitance was then used in the subsequent CapClamp.

An offline reexamination revealed that in several recorded cells the above fitting procedure yielded inaccurate estimates of the exponential components, e.g. very short fast components due to an artefactual voltage dip before pulse onset. To circumvent these problems, improved offline fits were performed for the artifact-free recharging at the pulse end (for more details see Appendix. In 8/18 cells, the offline and the original online estimate of the near capacitance differed by less than $20 \%$, but overall the offline measurement yielded higher capacitance values than originally used for the CapClamp (offline: $21.0 \pm 9.4 \mathrm{pF}$, online: $14.9 \pm 4.8 \mathrm{pF}$ ). In contrast to the online measurement, the offline procedure reported a better fit with three components for a subset of cells $(p<0.05,7 / 18)$, but for the analysis presented here the result of the two component fit is used in all cells.

Table 2: Multi-exponential fit and corresponding circuit parameters in the recorded dentate gyrus granule cells $(\mathrm{N}=18)$ and a multicompartment model based on a reconstructed DGGC morphology (see Simulations).

$$
\text { DGGCs (mean } \pm \text { std) multicomp. model }
$$

\section{Exp. fit}

$\tau_{0} \quad 15.1 \pm 4.8 \mathrm{~ms} \quad 15.1 \mathrm{~ms}$




\begin{tabular}{lll}
\hline & DGGCs (mean \pm std) & multicomp. model \\
\hline$R_{0}$ & $127.1 \pm 44.6 \mathrm{M} \Omega$ & $119.2 \mathrm{M} \Omega$ \\
$\tau_{1}$ & $0.77 \pm 0.24 \mathrm{~ms}$ & $0.18 \mathrm{~ms}$ \\
$R_{1}$ & $34.5 \pm 14.7 \mathrm{M} \Omega$ & $12.3 \mathrm{M} \Omega$ \\
Circuit & & \\
$C_{n}$ & $21.0 \pm 9.4 \mathrm{pF}$ & $13.0 \mathrm{pF}$ \\
$R_{n}$ & $854.2 \pm 394.0 \mathrm{M} \Omega$ & $1158.0 \mathrm{M} \Omega$ \\
$R_{a}$ & $52.5 \pm 19.8 \mathrm{M} \Omega$ & $15.5 \mathrm{M} \Omega$ \\
$C_{f}$ & $105.8 \pm 33.0 \mathrm{pF}$ & $113.7 \mathrm{pF}$ \\
$R_{f}$ & $155.5 \pm 59.9 \mathrm{M} \Omega$ & $132.8 \mathrm{M} \Omega$ \\
\hline
\end{tabular}

\subsubsection{Protocol 1: Verification of altered capacitance}

After online measurement of the capacitance, each DGGC was clamped at a range of capacitances from $60 \%$ to $300 \%$ of the original near capacitance. For each clamped capacitance, the above offline capacitance measurement protocol was repeated to see how the CapClamp altered the slow and fast components. These time scale and amplitude changes were then mapped to the corresponding two compartment circuit parameters to compare them to the target capacitance (see Measurement of near capacitance). Due to the difference between online and offline estimate of the original near capacitance, we corrected the original target capacitance to $C_{t}^{\text {corr }}=C_{c}^{\text {off }}+\Delta C_{t}$, which preserves the targeted capacitance change $\Delta C_{t}=C_{t}-C_{c}^{\text {on }}$. Equally, the clamping factors in the mapping were updated to $k=\frac{C_{t}^{\text {corr }}}{C_{c}^{\text {off }}}$.

\subsubsection{Protocol 2: Analysis of fI curves and spike shapes}

In a subset of cells, after measuring near capacitance, an fI curve was obtained for the original capacitance and for target capacitances in the above range. Current pulses were $1 \mathrm{~s}$ long and repeated three times, at amplitudes ranging from $90 \%$ to $200 \%$ of an estimated rheobase. This rheobase was estimated by the first occurrence of spiking in response to a ramp (length: $5 \mathrm{~s}$, height: $250 \mathrm{pA}$ ). For a quantitative comparison, the resulting fI curves were fit by a square-root function

$$
f(I)=\Theta\left(I-I_{\text {rheo }}\right) \text { gain } \sqrt{I-I_{\text {rheo }}}
$$

which captured their type 1 firing with a continuous frequency-current relationship [34].

Spikes were detected as a minimum $10 \mathrm{mV}$ elevation over the average depolarization during the pulse. For the mean action potential (AP) shape, varying spike forms from the initial $(<300 \mathrm{~ms})$ part of the pulse were discarded. The extracted AP features were peak amplitude, threshold voltage and threshold time to peak (voltage derivative crossing $10 \mathrm{mV} / \mathrm{ms}$ ), height (difference between peak and threshold), temporal width at half of the height and fast afterhyperpolarization (fAHP; a voltage dip of $-0.5 \mathrm{mV}$ or larger within $10 \mathrm{~ms}$ after the spike). For threshold and 
fAHP detection, the spike shape was filtered with a digital 4th order Butterworth filter with critical frequencies $3.3 \mathrm{kHz}$, respectively $1 \mathrm{kHz}$.

To understand, how much changes in capacitance affect the action potential generating currents, we compared the recorded spikes with hypothetical ones obtained by assuming unaltered currents with respect to the original near capacitance. For a target capacitance $C_{t}$, this hypothetical spike is a scaled version of the original spike,

$$
V_{\text {hypo }}(t)=V_{c}\left(t_{0}\right)+\frac{C_{c}}{C_{t}}\left(V_{c}(t)-V_{c}\left(t_{0}\right)\right)
$$

where $V_{c}(t)$ is the spike form at the original cell capacitance $C_{c}$ and the initial time $t_{0}$ was chosen to be $t_{\text {spike }}-3 \mathrm{~ms}$ short before onset of the spike generating currents.

\subsection{Simulations of the CapClamp}

Simulations of neuron models coupled to the CapClamp were implemented using the neuron simulator Brian2 [81] and the CapClamp was realized using the Brian2 provided NetworkOperation that updated the clamp current every sampling interval using Equation 5 with zero delay between voltage sampling and current injection (for links to the available code see Appendix.

\subsubsection{Biophysical neuron model}

In order to test the CapClamp in the presence of active ionic conductances, a Wang-Buzsáki (WB) neuron, a single compartment model of hippocampal interneurons, was used [23]. Gating dynamics and peak conductances of the transient sodium current and the delayed rectifier potassium current were modeled as described earlier [13, Appendix A]. When the capacitance is varied, the WB neuron undergoes a well-characterized series of bifurcations; in particular it exhibits a saddle-node loop (SNL) bifurcation at $C_{m}=1.47 \frac{\mu \mathrm{F}}{\mathrm{cm}^{2}}$ [13, Fig. 6]. Here, the specific membrane capacitance was chosen as $C_{m}=0.75 \frac{\mathrm{uF}}{\mathrm{cm}^{2}} \frac{\mu \mathrm{F}}{\mathrm{cm}^{2}}$ and the membrane area was set to $A=20000 \mu \mathrm{m}^{2}$, so that the original cell capacitance was $150 \mathrm{pF}$. Simulations were performed with the second order Runge-Kutta method, a time step of $1 \mu \mathrm{s}$ and dynamic clamp loop frequencies up to $100 \mathrm{kHz}$. Analysis of spike shapes was performed in the same way as for the recorded cells.

\subsubsection{Multicompartment model of a dentate gyrus granule cell}

For a controlled test of the CapClamp in an electrotonically non-compact cell, a morphologically reconstruction of a recorded DGGC was used as the basis for a multicompartment simulation. Soma and the two dendritic trees had a total area of $14126 \mu \mathrm{m}^{2}$. The axon was removed for the simulation. Membrane properties were assumed to be uniform and chosen such that they reproduced the average values of the total capacitance and the membrane time constant observed in the experiments: $C_{m}=\frac{C_{n} \overline{+} C_{f}}{A} \approx 0.9 \frac{\mu \mathrm{F}}{\mathrm{cm}^{2}}$ and $R_{m}=\frac{\tau_{0}}{C_{m}} \approx 16800 \Omega \mathrm{cm}^{2}$. The axial resistivity was chosen as $R_{a}=300 \Omega \mathrm{cm}$. Simulations were performed with exponential Euler integration, a time step of $10 \mu \mathrm{s}$ and a dynamic 
bioRxiv preprint doi: https://doi.org/10.1101/2021.11.12.468368; this version posted December 9, 2021. The copyright holder for this preprint (which was not certified by peer review) is the author/funder, who has granted bioRxiv a license to display the preprint in perpetuity. It is made available under aCC-BY-NC 4.0 International license.

507 clamp sampling frequency of $20 \mathrm{kHz}$. Capacitance measurement and clamp procedure were the same as in the recorded 508 DGGCs. 


\section{Acknowlegdements}

This work was supported by the German Ministry of Education and Research (grant no. 01GQ1403), the Deutsche Forschungsgemeinschaft (DFG, German Research Foundation - Project numbers GRK 1589/2, EXC 257, FOR 2134) and the European Research Council (grant no. 864243). We thank Jan Benda and Lukas Sonnenberg for their dedicated support with dynamic clamp and fruitful discussions. We are grateful to Eve Marder and Ekaterina Morozova for being able to test the RTXI implementation of the CapClamp and apply the CapClamp in neurons of the crustacean stomatogastric ganglion. We thank Robert Gowers and Philipp Norton for valuable feedback on the manuscript.

\section{Competing interests}

The authors declare that no competing interests exist. 


\footnotetext{
${ }^{2} \mathrm{~A}$ Z-transform $X_{i} \stackrel{Z}{\rightarrow} \hat{X}(z)$ is linear and has the delay property $X_{i-1} \stackrel{Z}{\rightarrow} z^{-1} \hat{X}(z)$ [82, Table 13.2].
} 
The transfer function of the coupled system $H_{\text {cell+dyn }}(z)$ can then be compared with the one of the target system $H_{\text {target }}(z)$ (Fig. 6 B). Additionally, the frequency-dependent impedance can be retrieved from the transfer function by

$$
Z_{\text {cell }+ \text { dyn }}(f)=H_{\text {cell }+ \text { dyn }}\left(e^{i 2 \pi f \Delta t}\right) .
$$

\subsubsection{Transfer function of the CapClamp}

The Z-transform of the CapClamp filter can be read directly from the general form of the transfer function (Eq. 11) and the CapClamp feedback coefficients (Eq.9),

$$
F_{\text {dyn }}(z)=\frac{C_{c}-C_{t}}{C_{t}} \frac{C_{c}}{\Delta t} \frac{1-z^{-1}}{1+\frac{C_{c}-C_{t}}{C_{t}} z^{-1}} .
$$

\subsubsection{Transfer function of the RC circuit}

In an RC circuit, the dynamics of the voltage are

$$
C \frac{\mathrm{d} V}{\mathrm{~d} t}=-\frac{V}{R}+I
$$

Thus, in a single time step $\Delta t$, when the current is fixed, the voltage evolves as

$$
V(k \Delta t)=V((k-1) \Delta t) e^{-\frac{\Delta t}{\tau}}+R I\left(1-e^{-\frac{\Delta t}{\tau}}\right),
$$

where $\tau=R C$ is the time constant. Applying the Z-transform results in the transfer function

$$
H_{R C}(z)=R\left(1-e^{-\frac{\Delta t}{\tau}}\right) \frac{1}{z-e^{-\frac{\Delta t}{\tau}}},
$$

which is subsequently used as the cell's transfer function $H_{\text {cell }}(z)=H_{\mathrm{RC}}(z)$.

\subsubsection{Transfer function of the clamped RC circuit}

Introducing $K=\frac{C_{c}-C_{t}}{C_{t}}$ and $h_{c}=\frac{\Delta t}{\tau_{c}}$, the RC circuit (Eq. 15) and CapClamp (Eq. 14 transfer functions can be combined using Equation 12 to get the transfer function of the combined system

$$
H_{\text {cell }+ \text { dyn }}(z)=R\left(1-e^{-h_{c}}\right) \frac{z+K}{z^{2}+\left(K-e^{-h_{c}}-\frac{1}{h_{c}} K\left(1-e^{-h_{c}}\right)\right) z-K\left(e^{-h_{c}}-\frac{1}{h_{c}}\left(1-e^{-h_{c}}\right)\right)} .
$$

In comparison, the transfer function of the target $\mathrm{RC}$ circuit reads

$$
H_{\text {target }}(z)=R\left(1-e^{-h_{t}}\right) \frac{1}{z-e^{-h_{t}}}
$$


with $h_{t}=\frac{\Delta t}{\tau_{t}}=\frac{\Delta t}{R C_{t}}$ reflecting the different target capacitance.

Figure 1 - suppl. 1 compares the resulting impedances for decreased and increased capacitances. As discussed in the Results, the impedance amplitudes fit well up to a tenth of the dynamic clamp frequency. A closer look at the transfer function explains the fit at low frequencies and the deviations at higher frequencies.

Input resistance is preserved The input resistance is equal to the impedance at zero frequency, that is at $z=e^{i 2 \pi 0}=$ 1 , which for both coupled and target system is the original resistance,

$$
H_{\text {cell+dyn }}(1)=H_{\text {target }}(1)=R \text {. }
$$

Poles and zeros For a further comparison, poles and zeros of the transfer functions are calculated. To simplify the expressions, it is assumed that the time constant of the original and target circuits are much larger than the sampling interval, that is $h_{c} \ll 1$ and $h_{t} \ll 1$.

Target circuit The target circuit has no zero and a single pole located at

$$
p_{t}^{(1)}=e^{-h_{t}}=1-h_{t}+\ldots
$$

Capacitance clamped circuit The coupled system has one zero at

$$
r_{c}^{(1)}=-K=1-\frac{C_{c}}{C_{t}} .
$$

The clamped circuit has two poles at

$$
p_{c}^{(1)}=1-(1+K) h_{c}+\ldots
$$

and

$$
p_{c}^{(2)}=\frac{K}{2} h_{c}+\ldots
$$

Comparison of poles All poles and zeros for an RC circuit in its original state and clamped at decreased and increased capacitances are shown in Figure 6 The first pole of the clamped circuit coincides with the one of the target circuit: $p_{c}^{(1)}=1-\frac{C_{c}}{C_{c}} \frac{\Delta t}{R C_{c}}=1-h_{t}=p_{t}^{(1)}$. As these pole lies close to $z=1$, they determine the lower frequency response, which explains why the impedance amplitudes fit so well in this range.

In addition to moving the existent pole of the cell circuit to the one of the target circuit, the CapClamp creates an additional pole $p_{c}^{(2)} \approx \frac{h_{c}}{2}\left(\frac{C_{c}}{C_{t}}-1\right)$ and a new zero $r_{c}^{(1)}=1-\frac{C_{c}}{C_{t}}$. Thus, at an increased capacitance $C_{t}>C_{c}$, the new pole lies in the left half of the unit circle and thereby increases the impedance at higher frequencies. In contrast, at a decreased capacitance, the additional zero moves into the left half of the complex plane and thereby decreases the impedance at higher frequencies. 
A
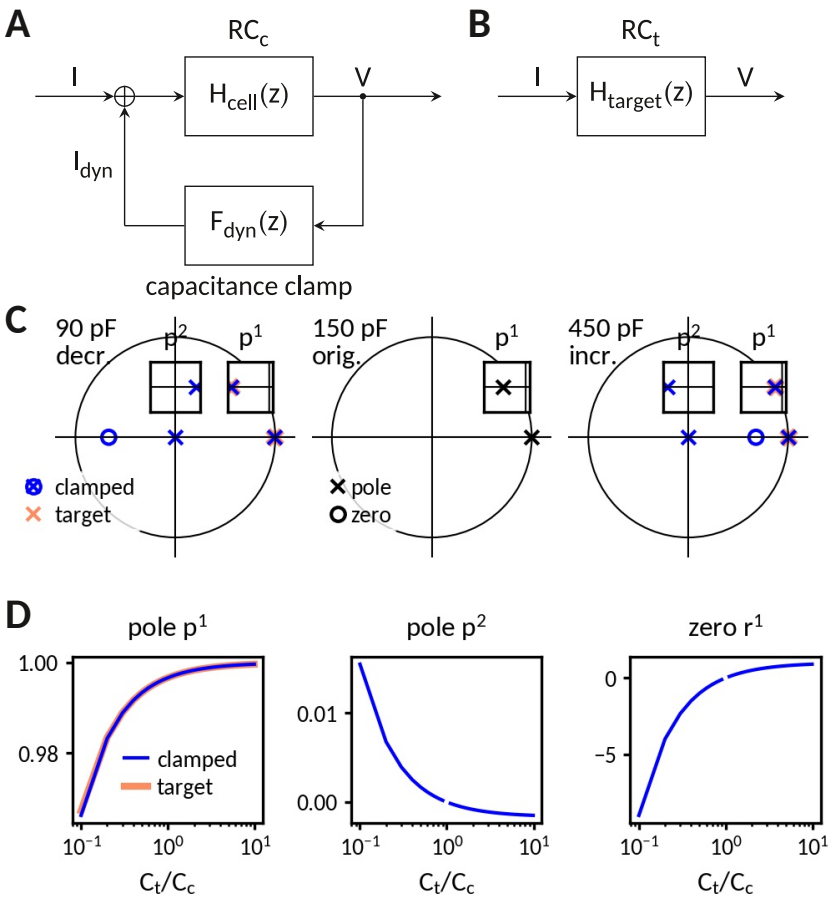

Figure 6: Analysis of the capacitance clamp as a discrete feedback filter. A Block diagram of the coupled system: RC circuit with original capacitance $C_{c}$ and capacitance clamp feedback current. B Block diagram of the target system: RC circuit with target capacitance $C_{t}$. C Pole-zero plot of the transfer functions at a decreased (left), the original and an increased capacitance. In addition to mimicking the pole of the target system, the clamped system has an additional pole and an additional zero. D Pole and zero position versus capacitance.

Stability For the investigated RC circuit with $R=100 \mathrm{M} \Omega$ and $C=150 \mathrm{pF}$ and a sampling interval of 50 us, both poles of the capacitance clamped system remain within the unit circle (Fig. 6) for the tested range from 0.1 to 10 times the original capacitance. As the coupled system is naturally causal, this implies that the transfer function of the clamped circuit is stable for this range of target capacitances, i.e. there are no unstable oscillations.

\subsection{Mapping between a charging curve with two components and a two compartment circuit}

In the following, we explain how a charging curve of a cell with two components can be mapped to the parameters of a two compartment circuit, which we used to extract the local capacitance in the recorded dentate gyrus granule cells (see Fig. 3). We first report the approach and results derived earlier [1, Appendix] and then explain how to extend the mapping when the capacitance is clamped to a modified value.

Golowasch et al. derived expressions for the near capacitance and the other circuit parameters by comparing the impedance of a two compartment circuit in Figure 3 A

$$
Z(s)=\frac{1}{\frac{1}{R_{n}}+s C_{n}+\frac{1}{R_{a}+\frac{1}{\frac{1}{R_{f}}+s C_{f}}}}
$$


with the impedance of a system whose response to a step currents is a sum of two exponentials

$$
Z(s)=R_{0} \frac{1}{1+s \tau_{0}}+R_{1} \frac{1}{1+s \tau_{1}}
$$

The comparison of these two impedances gives four equations linking the circuit parameters and the two components of the charging curve:

$$
\begin{aligned}
R_{0}+R_{1} & =\frac{R_{a} R_{n}+R_{f} R_{n}}{R_{a}+R_{f}+R_{n}}, \\
R_{0} \tau_{0}+R_{1} \tau_{1} & =\frac{R_{a} R_{n} R_{f} C_{f}}{R_{a}+R_{f}+R_{n}}, \\
\tau_{0}+\tau_{1} & =\frac{\left(R_{a}+R_{n}\right) C_{f} R_{f}+\left(R_{a}+R_{f}\right) C_{n} R_{n}}{R_{a}+R_{f}+R_{n}}, \\
\tau_{0} \tau_{1} & =\frac{R_{a} C_{n} R_{n} C_{f} R_{f}}{R_{a}+R_{f}+R_{n}} .
\end{aligned}
$$

To solve this set of equations, they assume that the membrane time constant is the same in all compartments $C_{n} R_{n}=$ $C_{f} R_{f}=\tau_{c}$. However in a clamped neuron, where the near capacitance is targeted to be modified to a k-fold different value, this equation becomes

$$
C_{n} R_{n}=k C_{f} R_{f}
$$

where $k=\frac{C_{n, \text { clam. }}}{C_{n, \text { orig. }}}$.

For the unclamped case, $k=1$, the mapping from the two components to the circuit parameters is

$$
\begin{aligned}
R_{n} & =R_{0}+\frac{\tau_{0}}{\tau_{1}} R_{1}, \\
C_{n} & =\frac{\tau_{0} \tau_{1}}{\tau_{1} R_{0}+\tau_{0} R_{1}}, \\
R_{f} & =\frac{R_{0} \tau_{1}}{R_{1} \tau_{0}}\left(R_{0}+\frac{\tau_{0}}{\tau_{1}} R_{1}\right) \\
C_{f} & =\frac{R_{1} \tau_{0}}{R_{0} \tau_{1}} \frac{\tau_{0} \tau_{1}}{\tau_{1} R_{0}+\tau_{0} R_{1}} \\
R_{a} & =\frac{\tau_{1}}{\tau_{0}-\tau_{1}}\left(R_{0}+\frac{\tau_{0}}{\tau_{1}} R_{1}\right)\left(1+\frac{R_{0} \tau_{1}}{R_{1} \tau_{0}}\right) .
\end{aligned}
$$

For the clamped case, $k \neq 1$, we used the python package sympy to solve the equations.

\subsection{Adapted fitting procedure of dentate gyrus charging curves}

The initial online capacitance measurement was based on fitting the charging curve at the beginning of the current pulse. Posterior analysis showed an artefactual voltage drop of $-0.2 \mathrm{mV}$ starting about $0.2 \mathrm{~ms}$ before pulse onset (probably due 
to coupling of the DAQ measurement card and the motherboard of the dynamic clamp computer), which limited the reliability of the online fit for cells with a small fast component. As no such artifact was observed for the recharging at the end of the pulse, this part was used in an improved offline fit. Additional measures to improve the fit were: cut of the first $0.2 \mathrm{~ms}$ after pulse end to minimize electrode artifacts, limiting the fit to the first $60 \mathrm{~ms}$ (3-4 times $\left.\tau_{0}\right)$ after the pulse to prioritize the early part of the charging curve and a switch to the python package lmfit for better evaluation of parameter confidence bounds (Imfit.github.io). Furthermore, the finite rise time of the current injection by the amplifier was taken into account by adapting the original form of the charging curve (Eq. 6) to

$$
\begin{aligned}
V(t)=I_{e x t} & {\left[\sum_{i ; \tau_{i} \neq \tau_{a}} \frac{R_{i}}{\tau_{i}-\tau_{a}}\left(\tau_{i}\left(1-e^{-\frac{t}{\tau_{i}}}\right)-\tau_{a}\left(1-e^{-\frac{t}{\tau_{a}}}\right)\right)+\right.} \\
& \left.\sum_{i ; \tau_{i}=\tau_{a}} R_{i}\left(1-e^{-\frac{t}{\tau_{i}}}-\frac{t}{\tau_{i}} e^{-\frac{t}{\tau_{i}}}\right)\right]
\end{aligned}
$$

where the current rise time of the amplifier $\tau_{a}(87 \pm 2 \mu \mathrm{s})$ was obtained by fitting the recorded injected current for the current step command by a simple exponential. A comparison of the two exponential components and the resulting circuit parameters for the online and offline fitting procedures is shown in Tab. 3 .

For the charging curves under capacitance clamp, the fitting procedure for the charging curve with two exponentials was initialized with values as expected for the targeted capacitance change: mapping the fitting results of the unclamped response to a two compartment circuit, changing the near capacitance to the targeted value and finally mapping this altered circuit back to the expected time scale and amplitudes. This initialization improved the fits especially at increased near capacitances, where the amplitude of the fast component becomes smaller.

Table 3: Comparison of online and offline fits to charging curves in the recorded dentate gyrus granule cells $(\mathrm{N}=18)$.

\begin{tabular}{lll}
\hline & online fit $($ mean \pm std $)$ & offline fit (mean $\pm \mathrm{stc})$ \\
\hline Two comp. & & \\
$\tau_{0}$ & $14.9 \pm 4.8 \mathrm{~ms}$ & $15.1 \pm 4.8 \mathrm{~ms}$ \\
$R_{0}$ & $136.9 \pm 47.5 \mathrm{M} \Omega$ & $127.1 \pm 44.6 \mathrm{M} \Omega$ \\
$\tau_{1}$ & $0.41 \pm 0.23 \mathrm{~ms}$ & $0.77 \pm 0.24 \mathrm{~ms}$ \\
$R_{1}$ & $25.1 \pm 14.1 \mathrm{M} \Omega$ & $34.5 \pm 14.7 \mathrm{M} \Omega$ \\
Circuit & & \\
$C_{n}$ & $14.9 \pm 4.7 \mathrm{pF}$ & $21.0 \pm 9.4 \mathrm{pF}$ \\
$R_{n}$ & $1106.3 \pm 519.3 \mathrm{M} \Omega$ & $854.2 \pm 394.0 \mathrm{M} \Omega$ \\
$R_{a}$ & $34.9 \pm 19.9 \mathrm{M} \Omega$ & $52.5 \pm 19.8 \mathrm{M} \Omega$ \\
$C_{f}$ & $99.1 \pm 33.7 \mathrm{pF}$ & $105.8 \pm 33.0 \mathrm{pF}$
\end{tabular}




\begin{tabular}{lll}
\hline & online fit $($ mean \pm std $)$ & offline fit $($ mean \pm std $)$ \\
\hline$R_{f}$ & $159.6 \pm 58.1 \mathrm{M} \Omega$ & $155.5 \pm 59.9 \mathrm{M} \Omega$ \\
\hline
\end{tabular}

\subsection{Data and software availability}

- Electrophysiological recordings of capacitance clamped dentate gyrus granule cells: Paul Pfeiffer, \& Federico José Barreda Tomás. (2021). Capacitance clamp demonstration in rat dentate gyrus granule cells (1.0.0) [Data set]. Zenodo. 10.5281/zenodo.5552207

- Project repository with capacitance clamp module for RELACS and custom analysis/simulation in python: Paul Pfeiffer, Federico José Barreda Tomás, Jiameng Wu, Jan-Hendrik Schleimer, Imre Vida, \& Susanne Schreiber. (2021). Softfware for: A dynamic clamp protocol to artificially modify cell capacitance (v1.0). Zenodo. 10.5281/zenodo.5762092

- Capacitance clamp plugin for RTXI, a real-time data-acquistion and control application for biological research that allows to extend a conventional electrophysiology setup for dynamic clamp experiments [51]: capacitance_clamp_rtxi_module: (v1.0.1). Zenodo. 10.5281/zenodo.5553946

\subsection{Supplementary figures}

- Figure 1 - suppl. 1; Impedance analysis of an RC circuit coupled to the capacitance clamp. 
A
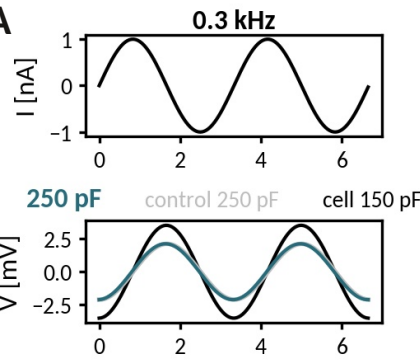

$100 \mathrm{pF}$ control $100 \mathrm{pF} \quad$ cell $150 \mathrm{pF}$
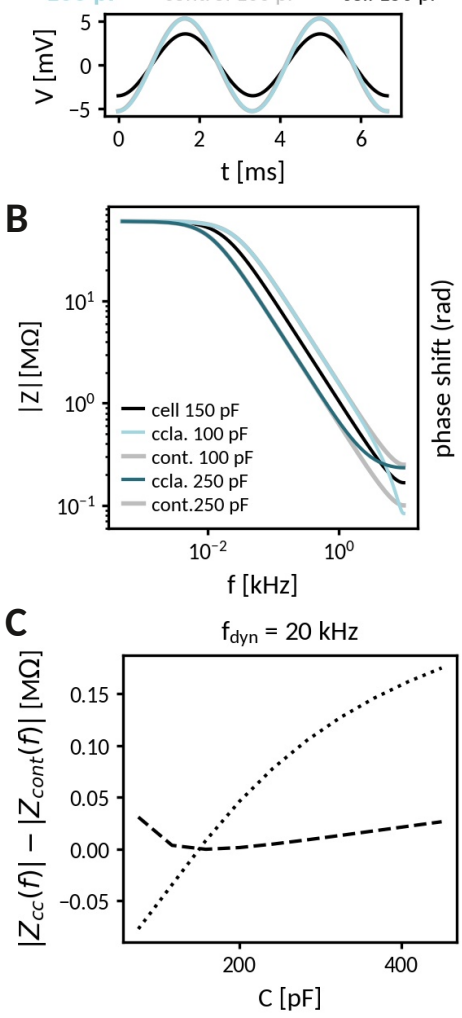
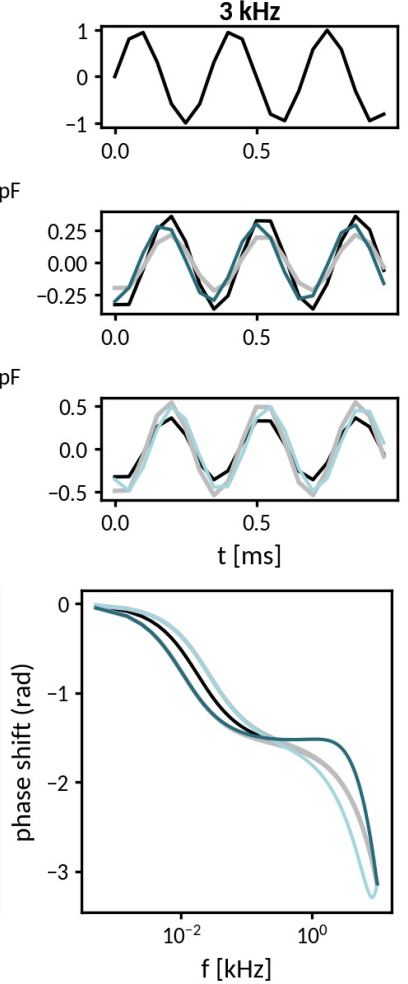

$\mathrm{f}[\mathrm{kHz}]$

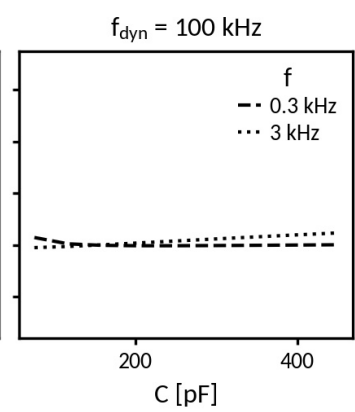

Figure 1 - suppl. 1: Impedance analysis of an RC circuit coupled to the capacitance clamp. A Injection of an oscillating current at $300 \mathrm{~Hz}$ (left) and at $3 \mathrm{kHz}$ (right) to a passive cell (RC-circuit) with voltage responses clamped at an increased (middle) and a decreased capacitance (bottom). Black lines indicate the response of the cell at the original capacitance and gray lines those of the corresponding control cells. B Comparison of frequency-dependent impedance and phase shift of a cell at the above capacitances (black: cell capacitance, blues: clamped, gray: control). C Difference of impedances at $300 \mathrm{~Hz}$ (dotted) and $3 \mathrm{kHz}$ (dashed) for clamped and control cell across different capacitances and for dynamic clamp frequencies of $20 \mathrm{kHz}$ (left) and $100 \mathrm{kHz}$ (right). 


\section{References}

[1] J. Golowasch, G. Thomas, A. L. Taylor, A. Patel, A. Pineda, C. Khalil, and F. Nadim. Membrane Capacitance Measurements Revisited: Dependence of Capacitance Value on Measurement Method in Nonisopotential Neurons. Journal of Neurophysiology, 102(4):2161-2175, 2009. ISSN 0022-3077. doi 10.1152/jn.00160.2009.

[2] W. E. White and S. L. Hooper. Contamination of current-clamp measurement of neuron capacitance by voltage-dependent phenomena. Journal of Neurophysiology, 110(1):257-268, 2013. ISSN 0022-3077. doi:10.1152/jn.00993.2012.

[3] Luc J. Gentet, Greg J. Stuart, and John D. Clements. Direct Measurement of Specific Membrane Capacitance in Neurons. Biophysical Journal, 79(1):314-320, July 2000. ISSN 00063495. doi:10.1016/S0006-3495(00)76293-X

[4] D.K. Hartline and D.R. Colman. Rapid Conduction and the Evolution of Giant Axons and Myelinated Fibers. Current Biology, 17(1):R29-R35, January 2007. ISSN 09609822. doi $10.1016 /$ j.cub.2006.11.042.

[5] R. Douglas Fields. White matter in learning, cognition and psychiatric disorders. Trends in Neurosciences, 31(7): 361-370, July 2008. ISSN 01662236. doi:10.1016/j.tins.2008.04.001.

[6] Guy Eyal, Matthijs B. Verhoog, Guilherme Testa-Silva, Yair Deitcher, Johannes C. Lodder, Ruth BenavidesPiccione, Juan Morales, Javier Defelipe, Christiaan P.J. de Kock, Huibert D. Mansvelder, and Idan Segev. Unique membrane properties and enhanced signal processing in human neocortical neurons. eLife, 5:1-18, 2016. ISSN 2050084X. doi:10.7554/eLife.16553.

[7] Lou Beaulieu-Laroche, Enrique H.S. Toloza, Marie-Sophie van der Goes, Mathieu Lafourcade, Derrick Barnagian, Ziv M. Williams, Emad N. Eskandar, Matthew P. Frosch, Sydney S. Cash, and Mark T. Harnett. Enhanced Dendritic Compartmentalization in Human Cortical Neurons. Cell, 175(3):643-651.e14, October 2018. ISSN 00928674. doi:10.1016/j.cell.2018.08.045

[8] Hugh P.C. Robinson. Conductance injection. Trends in Neurosciences, 17(4):147, January 1994. ISSN 01662236. doi:10.1016/0166-2236(94)90088-4

[9] Andrew A. Sharp, Michael B. O’Neil, L. F. Abbott, and Eve Marder. The dynamic clamp: Artificial conductances in biological neurons. Trends in Neurosciences, 16(10):389-394, 1993. ISSN 01662236. doi:10.1016/01662236(93)90004-6.

[10] David B. Jaffe and Robert Brenner. A computational model for how the fast afterhyperpolarization paradoxically increases gain in regularly firing neurons. Journal of Neurophysiology, 119(4):1506-1520, April 2018. ISSN 0022-3077, 1522-1598. doi:10.1152/jn.00385.2017.

[11] Alessio Franci, Guillaume Drion, and Rodolphe Sepulchre. Robust and tunable bursting requires slow positive feedback. Journal of Neurophysiology, 119(3):1222-1234, March 2018. ISSN 0022-3077, 1522-1598. doi:10.1152/jn.00804.2017. 
[12] Christoph Kirst, Julian Ammer, Felix Felmy, Andreas Herz, and Martin Stemmler. Fundamental Structure and Modulation of Neuronal Excitability: Synaptic Control of Coding, Resonance, and Network Synchronization. BioRxiv, July 2015. doi:10.1101/022475

[13] Janina Hesse, Jan Hendrik Schleimer, and Susanne Schreiber. Qualitative changes in phase-response curve and synchronization at the saddle-node-loop bifurcation. Physical Review E, 95(5), 2017. ISSN 24700053. doi:10.1103/PhysRevE.95.052203

[14] Susana Andrea Contreras, Jan-Hendrik Schleimer, Allan T. Gulledge, and Susanne Schreiber. Activity-mediated accumulation of potassium induces a switch in firing pattern and neuronal excitability type. BioRxiv, November 2020. doi $10.1101 / 2020.11 .30 .403782$

[15] Astrid A. Prinz, L. F. Abbott, and Eve Marder. The dynamic clamp comes of age. Trends in Neurosciences, 27(4): 218-224, 2004. ISSN 01662236. doi:10.1016/j.tins.2004.02.004

[16] M. N. Economo, F. R. Fernandez, and J. A. White. Dynamic Clamp: Alteration of Response Properties and Creation of Virtual Realities in Neurophysiology. Journal of Neuroscience, 30(7):2407-2413, 2010. ISSN 0270-6474. doi:10.1523/jneurosci.5954-09.2010.

[17] Gina G Turrigiano, Eve Marder, and L F Abbott. Cellular short-term memory from a slow potassium conductance. Journal of Neurophysiology, 75(2):963-6, 1996. ISSN 0022-3077. doi 10.1152/jn.1996.75.2.963

[18] Gytis Svirskis, Vibhakar Kotak, Dan H Sanes, and John Rinzel. Sodium Along With Low-Threshold Potassium Currents Enhance Coincidence Detection of Subthreshold Noisy Signals in MSO Neurons. Journal of Neurophysiology, 91:9, 2004. doi:10.1152/jn.00717.2003

[19] Steven A. Prescott, Stéphanie Ratté, Yves De Koninck, and Terrence J. Sejnowski. Pyramidal Neurons Switch From Integrators In Vitro to Resonators Under In Vivo-Like Conditions. Journal of Neurophysiology, 100(6): 3030-3042, December 2008. ISSN 0022-3077, 1522-1598. doi:10.1152/jn.90634.2008

[20] A. Hasenstaub, S. Otte, E. Callaway, and T. J. Sejnowski. Metabolic cost as a unifying principle governing neuronal biophysics. Proceedings of the National Academy of Sciences, 107(27):12329-12334, July 2010. ISSN 0027-8424, 1091-6490. doi:10.1073/pnas.0914886107.

[21] Attila Szúcs, Anikó Rátkai, Katalin Schlett, and Ramon Huerta. Frequency-dependent regulation of intrinsic excitability by voltage-activated membrane conductances, computational modeling and dynamic clamp. European Journal of Neuroscience, 46(9):2429-2444, 2017. ISSN 14609568. doi:10.1111/ejn.13708.

[22] Paul Pfeiffer, Alexei V Egorov, Franziska Lorenz, Jan-Hendrik Schleimer, Andreas Draguhn, and Susanne Schreiber. Clusters of cooperative ion channels enable a membrane-potential-based mechanism for short-term memory. eLife, 9:e49974, February 2020. ISSN 2050-084X. doi:10.7554/eLife.49974.

[23] Xiao-Jing Wang and György Buzsáki. Gamma Oscillation by Synaptic Inhibition in a Hippocampal Interneuronal Network Model. Journal of Neuroscience, 16(20):6402-6413, October 1996. ISSN 0270-6474, 1529-2401. doi:10.1523/JNEUROSCI.16-20-06402.1996. 
[24] Laura L. Rihn and Brenda J. Claiborne. Dendritic growth and regression in rat dentate granule cells during late postnatal development. Developmental Brain Research, 54(1):115-124, June 1990. ISSN 01653806. doi:10.1016/0165-3806(90)90071-6

[25] C. Schmidt-Hieber, P. Jonas, and J. Bischofberger. Subthreshold Dendritic Signal Processing and Coincidence Detection in Dentate Gyrus Granule Cells. Journal of Neuroscience, 27(31):8430-8441, August 2007. ISSN 0270-6474, 1529-2401. doi:10.1523/JNEUROSCI.1787-07.2007

[26] Willem A. M. Wybo, Benjamin Torben-Nielsen, Thomas Nevian, and Marc-Oliver Gewaltig. Electrical Compartmentalization in Neurons. Cell Reports, 26(7):1759-1773.e7, February 2019. ISSN 2211-1247. doi:10.1016/j.celrep.2019.01.074.

[27] Roland Krueppel, Stefan Remy, and Heinz Beck. Dendritic Integration in Hippocampal Dentate Granule Cells. Neuron, 71(3):512-528, August 2011. ISSN 08966273. doi:10.1016/j.neuron.2011.05.043

[28] Jegath C Athilingam, Roy Ben-Shalom, Caroline M Keeshen, Vikaas S Sohal, and Kevin J Bender. Serotonin enhances excitability and gamma frequency temporal integration in mouse prefrontal fast-spiking interneurons. eLife, 6:e31991, December 2017. ISSN 2050-084X. doi 10.7554/eLife.31991

[29] Go Ashida, Kousuke Abe, Kazuo Funabiki, and Masakazu Konishi. Passive Soma Facilitates Submillisecond Coincidence Detection in the Owl's Auditory System. Journal of Neurophysiology, 97(3):2267-2282, March 2007. ISSN 0022-3077, 1522-1598. doi:10.1152/jn.00399.2006

[30] Simon B. Laughlin, Rob R. de Ruyter van Steveninck, and John C. Anderson. The metabolic cost of neural information. Nature Neuroscience, 1(1):36-41, May 1998. ISSN 1097-6256, 1546-1726. doi:10.1038/236

[31] Bhanu P. Tewari, Lata Chaunsali, Susan L. Campbell, Dipan C. Patel, Adam E. Goode, and Harald Sontheimer. Perineuronal nets decrease membrane capacitance of peritumoral fast spiking interneurons in a model of epilepsy. Nature Communications, 9(1):4724, December 2018. ISSN 2041-1723. doi:10.1038/s41467-018-07113-0.

[32] Klaus M. Stiefel, Boris S. Gutkin, and Terrence J. Sejnowski. Cholinergic Neuromodulation Changes Phase Response Curve Shape and Type in Cortical Pyramidal Neurons. PLOS ONE, 3(12):e3947, December 2008. ISSN 1932-6203. doi:10.1371/journal.pone.0003947.

[33] Klaus M. Stiefel, Boris S. Gutkin, and Terrence J. Sejnowski. The effects of cholinergic neuromodulation on neuronal phase-response curves of modeled cortical neurons. Journal of Computational Neuroscience, 26(2): 289-301, April 2009. ISSN 0929-5313, 1573-6873. doi:10.1007/s10827-008-0111-9.

[34] Eugene M. Izhikevich. Dynamical Systems in Neuroscience: The Geometry of Excitability and Bursting. The MIT Press, 2006. ISBN 978-0-262-27607-8. doi:10.7551/mitpress/2526.001.0001.

[35] Steven A. Prescott, Yves De Koninck, and Terrence J. Sejnowski. Biophysical Basis for Three Distinct Dynamical Mechanisms of Action Potential Initiation. PLOS Computational Biology, 4(10):e1000198, October 2008. ISSN 1553-7358. doi:10.1371/journal.pcbi.1000198 
[36] Ronald Wilders. Dynamic clamp: A powerful tool in cardiac electrophysiologys. The Journal of Physiology, 576 (2):349-359, October 2006. ISSN 00223751. doi $10.1113 /$ jphysiol.2006.115840

[37] J. M. Bekkers and M. Hausser. Targeted dendrotomy reveals active and passive contributions of the dendritic tree to synaptic integration and neuronal output. Proceedings of the National Academy of Sciences, 104(27): 11447-11452, July 2007. ISSN 0027-8424, 1091-6490. doi:10.1073/pnas.0701586104

[38] Miklos Szoboszlay, Andrea Lórincz, Frederic Lanore, Koen Vervaeke, R. Angus Silver, and Zoltan Nusser. Functional Properties of Dendritic Gap Junctions in Cerebellar Golgi Cells. Neuron, 90(5):1043-1056, June 2016. ISSN 08966273. doi:10.1016/j.neuron.2016.03.029

[39] Jia Liu, Yoon Seok Kim, Claire E. Richardson, Ariane Tom, Charu Ramakrishnan, Fikri Birey, Toru Katsumata, Shucheng Chen, Cheng Wang, Xiao Wang, Lydia-Marie Joubert, Yuenwen Jiang, Huiliang Wang, Lief E. Fenno, Jeffrey B.-H. Tok, Sergiu P. Paşca, Kang Shen, Zhenan Bao, and Karl Deisseroth. Genetically targeted chemical assembly of functional materials in living cells, tissues, and animals. Science, 367(6484):1372, March 2020. doi: $10.1126 /$ science.aay 4866

[40] Bernardo L. de Oliveira, Emily R. Pfeiffer, Joakim Sundnes, Samuel T. Wall, and Andrew D. McCulloch. Increased Cell Membrane Capacitance is the Dominant Mechanism of Stretch-Dependent Conduction Slowing in the Rabbit Heart: A Computational Study. Cellular and Molecular Bioengineering, 8(2):237-246, June 2015. ISSN 1865-5025, 1865-5033. doi:10.1007/s12195-015-0384-9.

[41] Hillel Ori, Hananel Hazan, Eve Marder, and Shimon Marom. Dynamic clamp constructed phase diagram for the Hodgkin and Huxley model of excitability. Proceedings of the National Academy of Sciences, 117(7):3575-3582, February 2020. ISSN 0027-8424, 1091-6490. doi:10.1073/pnas.1916514117

[42] Janina Hesse and Susanne Schreiber. Externalization of neuronal somata as an evolutionary strategy for energy economization. Current Biology, 25(8):R324-R325, 2015. ISSN 09609822. doi $10.1016 /$ j.cub.2015.02.024.

[43] D H Edwards and B Mulloney. Compartmental models of electrotonic structure and synaptic integration in an identified neurone. The Journal of Physiology, 348(1):89-113, March 1984. ISSN 00223751. doi:10.1113/jphysiol.1984.sp015101.

[44] Willem AM Wybo, Jakob Jordan, Benjamin Ellenberger, Ulisses Marti Mengual, Thomas Nevian, and Walter Senn. Data-driven reduction of dendritic morphologies with preserved dendro-somatic responses. eLife, 10: e60936, January 2021. ISSN 2050-084X. doi 10.7554/eLife.60936.

[45] A. Norenberg, H. Hu, I. Vida, M. Bartos, and P. Jonas. Distinct nonuniform cable properties optimize rapid and efficient activation of fast-spiking GABAergic interneurons. Proceedings of the National Academy of Sciences, 107(2):894-899, January 2010. ISSN 0027-8424, 1091-6490. doi:10.1073/pnas.0910716107.

[46] Laurent Badel, Sandrine Lefort, Romain Brette, Carl C H Petersen, Wulfram Gerstner, and Magnus J E Richardson. Dynamic I-V Curves Are Reliable Predictors of Naturalistic Pyramidal-Neuron Voltage Traces. J Neurophysiol, 99:11, 2008. 
[47] Alan D Dorval, David J Christini, and John A White. Real-Time Linux Dynamic Clamp: A Fast and Flexible Way to Construct Virtual Ion Channels in Living Cells. Annals of Biomedical Engineering, 29:11, 2001.

[48] Jan Benda, Tim Gollisch, Christian K Machens, and Andreas VM Herz. From response to stimulus: Adaptive sampling in sensory physiology. Current Opinion in Neurobiology, 17(4):430-436, August 2007. ISSN 09594388. doi:10.1016/j.conb.2007.07.009

[49] Ildikó Kemenes, Vincenzo Marra, Michael Crossley, Dávid Samu, Kevin Staras, György Kemenes, and Thomas Nowotny. Dynamic clamp with StdpC software. Nature Protocols, 6(3):405-417, March 2011. ISSN 1750-2799. doi: $10.1038 /$ nprot.2010.200

[50] Daniele Linaro, João Couto, and Michele Giugliano. Real-time Electrophysiology: Using Closed-loop Protocols to Probe Neuronal Dynamics and Beyond. Journal of Visualized Experiments, (100):52320, June 2015. ISSN 1940-087X. doi: $10.3791 / 52320$

[51] Yogi A. Patel, Ansel George, Alan D. Dorval, John A. White, David J. Christini, and Robert J. Butera. Hard real-time closed-loop electrophysiology with the Real-Time eXperiment Interface (RTXI). PLOS Computational Biology, 13(5):e1005430, 2017. ISSN 1553-7358. doi/10.1371/journal.pcbi.1005430

[52] Niraj S. Desai, Richard Gray, and Daniel Johnston. A Dynamic Clamp on Every Rig. eneuro, 4(5):ENEURO.025017.2017, September 2017. ISSN 2373-2822. doi 10.1523/ENEURO.0250-17.2017.

[53] Rodrigo Amaducci, Manuel Reyes-Sanchez, Irene Elices, Francisco B. Rodriguez, and Pablo Varona. RTHybrid: A Standardized and Open-Source Real-Time Software Model Library for Experimental Neuroscience. Frontiers in Neuroinformatics, 13:11, March 2019. ISSN 1662-5196. doi:10.3389/fninf.2019.00011.

[54] Jonathan C. Bettencourt, Kyle P. Lillis, Laura R. Stupin, and John A. White. Effects of imperfect dynamic clamp: Computational and experimental results. Journal of Neuroscience Methods, 169(2):282-289, 2008. ISSN 08966273. doi:10.1016/j.jneumeth.2007.10.009

[55] Romain Brette, Zuzanna Piwkowska, Cyril Monier, Michelle Rudolph-Lilith, Julien Fournier, Manuel Levy, Yves Frégnac, Thierry Bal, and Alain Destexhe. High-Resolution Intracellular Recordings Using a RealTime Computational Model of the Electrode. Neuron, 59(3):379-391, August 2008. ISSN 08966273. doi:10.1016/j.neuron.2008.06.021

[56] Thierry Bal and Alain Destexhe, editors. Dynamic-Clamp: From Principles to Applications. Springer US, New York, NY, 2009. ISBN 978-0-387-89278-8 978-0-387-89279-5. doi:10.1007/978-0-387-89279-5

[57] Florin Amzica and Dag Neckelmann. Membrane Capacitance of Cortical Neurons and Glia During Sleep Oscillations and Spike-Wave Seizures. Journal of Neurophysiology, 82(5):2731-2746, 1999. ISSN 0022-3077. doi:10.1152/jn.1999.82.5.2731

[58] Biswa Sengupta, Martin Stemmler, Simon B. Laughlin, and Jeremy E. Niven. Action Potential Energy Efficiency Varies Among Neuron Types in Vertebrates and Invertebrates. PLOS Computational Biology, 6(7):e1000840, July 2010. ISSN 1553-7358. doi 10.1371 /journal.pcbi.1000840 
[59] Ann M. Castelfranco and Daniel K. Hartline. The evolution of vertebrate and invertebrate myelin: A theoretical computational study. Journal of Computational Neuroscience, 38(3):521-538, June 2015. ISSN 0929-5313, 1573-6873. doi:10.1007/s10827-015-0552-x.

[60] William F Podlaski, Alexander Seeholzer, Lukas N Groschner, Gero Miesenböck, Rajnish Ranjan, and Tim P Vogels. Mapping the function of neuronal ion channels in model and experiment. eLife, 6:e22152, March 2017. ISSN 2050-084X. doi:10.7554/eLife.22152

[61] Nathan W. Gouwens, Jim Berg, David Feng, Staci A. Sorensen, Hongkui Zeng, Michael J. Hawrylycz, Christof Koch, and Anton Arkhipov. Systematic generation of biophysically detailed models for diverse cortical neuron types. Nature Communications, 9(1):710, December 2018. ISSN 2041-1723. doi:10.1038/s41467-017-02718-3.

[62] Mikhail G. Shapiro, Kazuaki Homma, Sebastian Villarreal, Claus Peter Richter, and Francisco Bezanilla. Infrared light excites cells by changing their electrical capacitance. Nature communications, 8:16148, 2017. ISSN 20411723. doi $10.1038 /$ ncomms 16148 .

[63] João L. Carvalho-de-Souza, Bernardo I. Pinto, David R. Pepperberg, and Francisco Bezanilla. Optocapacitive Generation of Action Potentials by Microsecond Laser Pulses of Nanojoule Energy. Biophysical Journal, 114(2): 283-288, 2018. ISSN 15420086. doi:10.1016/j.bpj.2017.11.018.

[64] B. Krasovitski, V. Frenkel, S. Shoham, and E. Kimmel. Intramembrane cavitation as a unifying mechanism for ultrasound-induced bioeffects. Proceedings of the National Academy of Sciences, 108(8):3258-3263, February 2011. ISSN 0027-8424, 1091-6490. doi:10.1073/pnas.1015771108

[65] Michael Plaksin, Shy Shoham, and Eitan Kimmel. Intramembrane Cavitation as a Predictive Bio-Piezoelectric Mechanism for Ultrasonic Brain Stimulation. Physical Review X, 4(1):011004, January 2014. ISSN 2160-3308. doi: $10.1103 /$ PhysRevX.4.011004.

[66] Timothy O’Leary, Alex H. Williams, Alessio Franci, and Eve Marder. Cell Types, Network Homeostasis, and Pathological Compensation from a Biologically Plausible Ion Channel Expression Model. Neuron, 82(4):809-821, 2014. ISSN 10974199. doi $10.1016 / j$.neuron.2014.04.002.

[67] Simone Temporal, Kawasi M. Lett, and David J. Schulz. Activity-Dependent Feedback Regulates Correlated Ion Channel mRNA Levels in Single Identified Motor Neurons. Current Biology, 24(16):1899-1904, August 2014. ISSN 09609822. doi:10.1016/j.cub.2014.06.067

[68] Joseph M. Santin and David J. Schulz. Membrane Voltage Is a Direct Feedback Signal That Influences Correlated Ion Channel Expression in Neurons. Current Biology, 29(10):1683-1688.e2, May 2019. ISSN 09609822. doi:10.1016/j.cub.2019.04.008

[69] Pablo Chamorro, Carlos Muñiz, Rafael Levi, David Arroyo, Francisco B. Rodríguez, and Pablo Varona. Generalization of the Dynamic Clamp Concept in Neurophysiology and Behavior. PLOS ONE, 7(7):e40887, July 2012. ISSN 1932-6203. doi:10.1371/journal.pone.0040887. 
[70] Ghanim Ullah and Steven J. Schiff. Tracking and control of neuronal Hodgkin-Huxley dynamics. Physical Review E - Statistical, Nonlinear, and Soft Matter Physics, 79(4):1-4, $2009 . \quad$ ISSN 15393755. doi:10.1103/PhysRevE.79.040901

[71] Catalina Rivera, Hyuck-Jin Kwon, Ali Hashmi, Gan Yu, Jiheng Zhao, Jianlong Gao, Jie Xu, Wei Xue, and Alexander Dimitrov. Towards a Dynamic Clamp for Neurochemical Modalities. Sensors, 15(5):10465-10480, May 2015. ISSN 1424-8220. doi:10.3390/s150510465.

[72] Patrick Harrigan, Hiten D. Madhani, and Hana El-Samad. Real-Time Genetic Compensation Defines the Dynamic Demands of Feedback Control. Cell, 175(3):877-886.e10, October 2018. ISSN 00928674. doi:10.1016/j.cell.2018.09.044

[73] Jonathan P Newman, Ming-fai Fong, Daniel C Millard, Clarissa J Whitmire, Garrett B Stanley, and Steve M Potter. Optogenetic feedback control of neural activity. eLife, 4:e07192, July 2015. ISSN 2050-084X. doi:10.7554/eLife.07192

[74] David Hocker and Il Memming Park. Myopic control of neural dynamics. PLOS Computational Biology, 15(3): e1006854, March 2019. ISSN 1553-7358. doi:10.1371/journal.pcbi.1006854.

[75] Dan Bar-Yehuda and Alon Korngreen. Space-Clamp Problems When Voltage Clamping Neurons Expressing Voltage-Gated Conductances. Journal of Neurophysiology, 99(3):1127-1136, March 2008. ISSN 0022-3077, 1522-1598. doi:10.1152/jn.01232.2007.

[76] Guy Major, Jonathan D Evans, and J Julian B Jack. Solutions for Transients in Arbitrarily Branching Cables: 1. Voltage Recording with a Somatic Shunt. Biophysical Journal, 65:27, 1993. doi:10.1016/S0006-3495(93)81037-3.

[77] Sam A. Booker, Jie Song, and Imre Vida. Whole-cell Patch-clamp Recordings from Morphologically- and Neurochemically-identified Hippocampal Interneurons. Journal of Visualized Experiments, (91):51706, September 2014. ISSN 1940-087X. doi:10.3791/51706.

[78] Linqing Feng, Ting Zhao, and Jinhyun Kim. neuTube 1.0: A New Design for Efficient Neuron Reconstruction Software Based on the SWC Format. eneuro, 2(1):ENEURO.0049-14.2014, January 2015. ISSN 2373-2822. doi:10.1523/ENEURO.0049-14.2014

[79] SciPy 1.0 Contributors, Pauli Virtanen, Ralf Gommers, Travis E. Oliphant, Matt Haberland, Tyler Reddy, David Cournapeau, Evgeni Burovski, Pearu Peterson, Warren Weckesser, Jonathan Bright, Stéfan J. van der Walt, Matthew Brett, Joshua Wilson, K. Jarrod Millman, Nikolay Mayorov, Andrew R. J. Nelson, Eric Jones, Robert Kern, Eric Larson, C J Carey, İlhan Polat, Yu Feng, Eric W. Moore, Jake VanderPlas, Denis Laxalde, Josef Perktold, Robert Cimrman, Ian Henriksen, E. A. Quintero, Charles R. Harris, Anne M. Archibald, Antônio H. Ribeiro, Fabian Pedregosa, and Paul van Mulbregt. SciPy 1.0: Fundamental algorithms for scientific computing in Python. Nature Methods, 17(3):261-272, March 2020. ISSN 1548-7091, 1548-7105. doi:10.1038/s41592-019-0686-2.

[80] W G Bardsley, P B McGinlay, and A J Wright. The F test for model discrimination with exponential functions. Biometrika, 73(2):8, 1986. doi $10.2307 / 2336228$. 
[81] Marcel Stimberg, Romain Brette, and Dan FM Goodman. Brian 2, an intuitive and efficient neural simulator. eLife, 8:e47314, August 2019. ISSN 2050-084X. doi 10.7554/eLife.47314.

[82] Richard Dorf and Robert Bishop. Modern Control Systems. Pearson, twelfth edition, 2010. ISBN 978-0-13602458-3. 